\title{
Galactic cosmic-ray propagation in the light of AMS-02: Analysis of protons, helium, and antiprotons
}

\author{
Michael Korsmeier ${ }^{*}$ and Alessandro Cuoco ${ }^{\dagger}$ \\ Institute for Theoretical Particle Physics and Cosmology, RWTH Aachen University, \\ 52056 Aachen, Germany
}

(Received 3 August 2016; published 30 December 2016)

\begin{abstract}
We present novel constraints on cosmic-ray propagation in the Galaxy using the recent precise measurements of proton and helium spectra from AMS-02, together with preliminary AMS-02 data on the antiproton over proton ratio. To explore efficiently the large (up to 11-dimensional) parameter space we employ the nested-sampling algorithm as implemented in the MULTINEST package, interfaced with the GALPROP code to compute the model-predicted spectra. We use VOYAGER proton and helium data, sampling the local interstellar spectra, to constrain the solar modulation potential. We find that the turbulence of the Galactic magnetic field is well constrained, i.e., $\delta=0.30_{-0.02}^{+0.03}$ (stat $)_{-0.04}^{+0.10}$ (sys), with uncertainties dominated by systematic effects. Systematic uncertainties are determined checking the robustness of the results to the minimum rigidity cut used to fit the data (from $1 \mathrm{GV}$ to $5 \mathrm{GV}$ ), to the propagation scenario (convection vs no convection), and to the uncertainties in the knowledge of the antiproton production cross section. Convection and reacceleration are found to be degenerate and not well constrained singularly when using data above $5 \mathrm{GV}$. Using data above $1 \mathrm{GV}$ reacceleration is required, $v_{\mathrm{A}}=25 \pm 2 \mathrm{~km} / \mathrm{s}$, although this value might be significantly affected by the low-energy systematic uncertainty in the solar modulation. In a forthcoming companion paper, we investigate the constraints imposed by AMS-02 measurements on lithium, boron, and carbon.
\end{abstract}

DOI: $10.1103 /$ PhysRevD.94.123019

\section{INTRODUCTION}

Cosmic-ray (CR) physics is on the verge of transition to a precision era thanks to the recently available data from PAMELA first, and more recently from the AMS-02 experiment on board the International Space Station. Thanks to these precise data, cracks in the standard minimal scenario start to appear. For example, a significant difference in the slopes of proton and helium, of about $\sim 0.1$ [1-3]), has been observed, while, from the standard CR acceleration scenario, no differences would be expected, at least for energies above $20-30 \mathrm{GeV}$. The same measurements also find a break in the proton and helium rigidity spectra at about $300 \mathrm{GV}$. In this case, the feature can be accommodated with an extension of the standard scenario, and various explanations have been proposed [4-8].

Nonetheless, besides the above CR "anomalies," the standard diffusion-reacceleration-convection scenario is, in the first place, not yet very well constrained. For example, estimates of the degree of turbulence in the Galactic magnetic field (encoded in the parameter conventionally indicated as $\delta$ ) range from the standard Kolmogorov turbulence $(\delta=0.33[9,10])$ to Kraichnan $(\delta=0.5)$ or plain diffusion $(\delta=0.6[11])$, up to $\delta=0.9[12,13]$. Again, the new precise data offer the possibility to finally pin down the uncertainties in the parameters of the model.

\footnotetext{
*korsmeier@physik.rwth-aachen.de

†cuoco@physik.rwth-aachen.de
}

In the following, we will thus analyze recently published proton [2] and helium [3] AMS-02 data together with preliminary AMS-02 data on the antiproton over proton ratio [14]. The analysis of the heavier nuclei, lithium, boron, and carbon and comparison with the results from the analysis of this work is presented in a companion forthcoming article.

We also treat in a novel way the effect of solar modulation. Although we still use the force-field approximation, we do not assume any prior on the solar modulation potential, but we, instead, use recent VOYAGER data [15], sampling the interstellar unmodulated CR spectrum, to constrain the amount of solar modulation. The effect of solar modulation will also be studied applying different cuts on the minimum rigidity of the data used in the fit. Finally, we will also investigate the effect of uncertainties in the antiproton production cross section, following the recent redetermination from [16].

There is another well-known anomaly in CRs, namely the rising positron fraction observed by both PAMELA [17] and AMS-02 [18]. The rising is incompatible with the usual interpretation of positrons as secondaries produced by protons during their propagation. Although some attempt has been made to reconcile the positron fraction with the interpretation as secondaries through some modification of the propagation model [19], the generally accepted explanation requires a primary source of positrons, like pulsars or a nearby supernova remnant, or possibly dark matter 
annihilation. In our study, we will assume the standard propagation scenario described above, and, as such, we would require a primary positron source to explain the observations. Nonetheless, even after including a primary source, it might be nontrivial to explain the positron fraction as well as the $e^{+}+e^{-}$spectrum, since the propagation of leptons is significantly affected by energy losses in the local radiation and magnetic field, while the local turbulence properties of the magnetic field can be different from the large scale average probed by nuclei. Although it would be, thus, interesting to cross-check the results of the study of propagation of nuclei with lepton spectra observations, ultimately, the above issues would make the comparison complicated and difficult to interpret. We will thus avoid these comparisons in the following and focus only on nuclei.

The work is structured as follows: The theoretical framework is discussed in Sec. II. The fit methodology is discussed in Sec. III. The results are presented in Sec. IV, while we conclude in Sec. V.

\section{THEORY}

The propagation of $\mathrm{CR}$ can be described by the wellknown diffusion equation [20] for the particle density $\psi_{i}$ of species $i$ per volume and absolute value of momentum $p$,

$$
\begin{aligned}
\frac{\partial \psi_{i}(\boldsymbol{x}, p, t)}{\partial t}= & q_{i}(\boldsymbol{x}, p)+\boldsymbol{\nabla} \cdot\left(D_{x x} \boldsymbol{\nabla} \psi_{i}-\boldsymbol{V} \psi_{i}\right) \\
& +\frac{\partial}{\partial p} p^{2} D_{p p} \frac{\partial}{\partial p} \frac{1}{p^{2}} \psi_{i} \\
& -\frac{\partial}{\partial p}\left(\frac{\mathrm{d} p}{\mathrm{~d} t} \psi_{i}-\frac{p}{3}(\boldsymbol{\nabla} \cdot \boldsymbol{V}) \psi_{i}\right) \\
& -\frac{1}{\tau_{f, i}} \psi_{i}-\frac{1}{\tau_{r, i}} \psi_{i} .
\end{aligned}
$$

The various terms describe (i) spatial diffusion, usually assumed to be homogeneous and isotropic and thus described by the momentum-dependent diffusion coefficient $D_{x x}(p)$, (ii) convective winds, described by their velocity $\boldsymbol{V}(\boldsymbol{x})$, (iii) diffusive reacceleration, parametrized as a diffusion in momentum space with coefficient $D_{p p}(p)$, (iv) continuous energy losses through the coefficient $d p / d t=\sum_{k} d p_{k} / d t$ which sums over all the various processes, $d p_{k} / d t$, through which the particles lose energy, (v) adiabatic energy losses, present if $\boldsymbol{V}(\boldsymbol{x})$ has a nonzero divergence, and finally, catastrophic losses by (vi) decay or (vii) fragmentation, with decay and interaction times $\tau_{r}$ and $\tau_{f}$, respectively. The equation is typically solved assuming a steady state regime, meaning that $\psi_{i}$ does not depend on time and so the term on the left-hand side is zero.

Diffusion is naturally expected to be an energy dependent process, with particles being less deflected by the magnetic fields with increasing energy, and thus diffusing faster. This process is usually modeled by a power law in rigidity $R=p /|Z|[21]$,

$$
D_{x x}=\beta D_{0}\left(\frac{R}{4 \mathrm{GV}}\right)^{\delta}
$$

where $\delta$ is the index of the power law, $D_{0}$ the overall normalization, and $\beta=v / c$ the velocity of the CRs; we set the normalization scale at $4 \mathrm{GV}$. The constant for diffusive reacceleration $D_{p p}$ is usually related to the spatial diffusion $D_{x x}$ and to the velocity $v_{\mathrm{A}}$ of Alfven magnetic waves [22,23] as

$$
D_{p p}=\frac{4\left(p v_{\mathrm{A}}\right)^{2}}{3(2-\delta)(2+\delta)(4-\delta) \delta D_{x x}} .
$$

The amount of reacceleration is thus described in terms of the parameter $v_{\mathrm{A}}$. Finally, convective winds are assumed to be constant and orthogonal to the Galactic plane $\boldsymbol{V}(\boldsymbol{x})=\operatorname{sign}(z) v_{0, c}$. We note that, in principle, this parametrization implies an unphysical discontinuity at $z=0$. A smooth transition in the thin halo containing the sources (with size $\sim 0.2 \mathrm{kpc}$ ) would be more realistic. Nonetheless, since this parametrization has been widely employed in past works, we use it for the sake of comparison.

The source term $q_{i}(\boldsymbol{x}, p)$ of primary $\mathrm{CR}$ is assumed to factorize into a species dependent normalization $q_{0, i}$, a space-depend part $q_{r, z}$ (where $r=\sqrt{x^{2}+y^{2}}$ and $z$ are galactocentric cylindrical coordinates), and a rigidity dependent part $q_{R}$,

$$
q_{i}(\boldsymbol{x}, p)=q_{i}(r, z, R)=q_{0, i} q_{r, z}(r, z) q_{R}(R) .
$$

We model the rigidity dependence as double broken power law with smooth transitions

$$
q_{R}(R)=\left(\frac{R}{R_{0}}\right)^{-\gamma_{1}}\left(\frac{R_{0}^{\frac{1}{s}}+R^{\frac{1}{s}}}{2\left(R_{0}\right)^{\frac{1}{s}}}\right)^{-s\left(\gamma_{2}-\gamma_{1}\right)}\left(\frac{R_{1}^{\frac{1}{s_{1}}}+R^{\frac{1}{s_{1}}}}{R_{1}^{\frac{1}{s_{1}}}}\right)^{-s_{1}\left(\gamma_{3}-\gamma_{2}\right)},
$$

where $R_{0}, R_{1}$ are the two break positions, $s, s_{1}$ the smoothing factors, and $\gamma_{i}(i=1,2,3)$ the slopes in the various rigidity ranges in between the breaks. The normalization is such that $q_{R}(R)=1$ at $R=R_{0}$. Typically, only one break has been considered in the literature, with value of the order $\sim 10 \mathrm{GV} \mathrm{[9]}$ or none ${ }^{1}$ [11]. On the other hand, the recent discovery of a break at around $300 \mathrm{GV}$ in the proton and helium spectra first by PAMELA [1] and then by AMS-02 [2,3] makes it necessary to introduce a

\footnotetext{
${ }^{1}$ In $[13,24]$ a source term $q \propto \beta^{-1} R^{-\gamma}$ is considered, which implies a break in momentum at a rigidity $\sim m / Z$, with an upward steepening of 1 in the slope.
} 
second break for a proper description of the data. This was, indeed, considered, for example, in [10,25]. We further introduced in Eq. (5), as a novel feature with respect to previous studies, the parameters $s_{i}$ to explore the possibility of a smooth transition between the various regimes, as opposed to a sharp one.

We mention here that an alternative possibility would be to model the break as a break in the diffusion rather than the injection spectrum. This has the same effect for the primaries' spectra but leads to different results for secondaries. The secondaries' injection spectra would reflect the break from the primary spectra, but the amount of the break would increase during the propagation, with the result that the break is expected to be twice as large as the one of primaries. Nonetheless, for antiprotons this effect would start to be significant only at very large energies (above few hundreds GV), which are not yet well measured by AMS-02, and thus the two scenarios are equivalent. The effect could be, instead, important for lithium or boron AMS-02 measurements, which extend to larger energies with respect to antiprotons.

The spatial dependence, i.e., the source distribution, is parametrized as

$$
q_{r, z}(r, z)=\left(\frac{r}{r_{s}}\right)^{\alpha} \exp \left(-\beta \frac{r-r_{s}}{r_{s}}\right) \exp \left(-\frac{|z|}{z_{0}}\right),
$$

with parameters $\alpha=0.5, \beta=1.0, r_{s}=8.5 \mathrm{kpc}$, and $z_{0}=0.2 \mathrm{kpc}$. For the analysis of $\gamma$ rays one usually uses source distribution inferred from pulsars [26] or supernova remnants $[27,28]$. Typical parameter values in those cases are $\alpha \sim 1.6, \beta \sim 4$ with a flattening above $r \gtrsim 10 \mathrm{kpc}$ and a cutoff above $r \gtrsim 30 \mathrm{kpc}$. We checked that changing the source distribution to those values has a negligible impact on the CR energy spectra after propagation.

In the case of secondary CRs, as for antiprotons produced by primary CRs through spallation in the interstellar medium (ISM), the source term is given by the primaries themselves. More precisely the source term is the integral over the momentum-dependent production rate of the secondaries and the sum over the primary species $i$ and the ISM components $j$,

$q(\boldsymbol{x}, p)=\sum_{j=\mathrm{H}, \mathrm{He}} n_{j}(\boldsymbol{x}) \sum_{i=p, \mathrm{He}} \int \mathrm{d} p_{i} \frac{\mathrm{d} \sigma_{i j}\left(p, p_{i}\right)}{\mathrm{d} p} \beta_{i} c \psi_{i}\left(\boldsymbol{x}, p_{i}\right)$,

where $\sigma_{i j}$ is the antiproton production cross section by the species $i$ spallating over the ISM species $j$. The ISM is assumed to be composed of hydrogen and helium gas with fixed proportion $1: 0.11$. The abundance of secondaries is typically quite low with respect to the primaries, and this allows one to evaluate Eq. (7) with $\psi_{j}\left(\boldsymbol{x}, p_{i}\right)$ calculated from Eq. (1) neglecting in the first place the secondaries.
Besides antiprotons, we will consider also secondary protons, i.e., primary protons that underwent inelastic scattering, losing a substantial fraction of their energy, and thus reappearing at low energies. We will also take into account tertiary antiprotons produced by the spallation of the secondary antiprotons during propagation. Secondary protons and tertiary antiprotons are described with the same formalism. Their source term can be calculated analogously to Eq. (7) but replacing $\psi_{i}\left(\boldsymbol{x}, p_{i}\right)$ with the density of primary protons in the first case, and secondary antiprotons in the second case, and using the associated production cross section. The latter is approximated as the total inelastic nonannihilating cross section of the incoming proton or antiproton times the energy distribution of the scattered particle, approximated as $1 / E_{\mathrm{kin}}$. For more details see Ref. [29].

To numerically solve the propagation equation Eq. (1) and to derive the secondaries' and tertiaries' abundances we use the GALPROP code ${ }^{2}[30,31]$. We use version $r 2766^{3}$ as the basis, and we implement some custom modifications, such as the possibility to use species-dependent injection spectra, which is not allowed by default in GALPROP. Furthermore, we allow for a smoothing of the originally simple broken power law as discussed above.

The propagation equation (1) is solved on a grid in the energy dimension and in the two spatial dimensions $r$ and $z$, assuming cylindrical symmetry of our Galaxy. The radial boundary of the Galaxy is fixed to $20 \mathrm{kpc}$, while the halfheight $z_{h}$ is a free parameter. The radial and $z$ grid steps are chosen as $\Delta r=1 \mathrm{kpc}$ and $\Delta z=0.2 \mathrm{kpc}$. The grid in kinetic energy per nucleon is logarithmic between 1 and $10^{7} \mathrm{MeV}$ with a step factor of 1.4. Free escape boundary conditions are used, imposing $\psi_{i}$ equal to zero outside the region sampled by the grid. We tested also more accurate choices for the above settings and found the results stable against the changes.

Note also that we consider propagation of nuclei only up to $Z=2$; i.e., in practice, in GALPROP we propagate $p, \bar{p}$, ${ }^{2} \mathrm{H},{ }^{3} \mathrm{He}$, and ${ }^{4} \mathrm{He}$ species plus the secondary protons and the tertiaries' antiprotons. This also means that we neglect possible contributions from the fragmentation of $Z>2$ nuclei, which should be a good approximation since their fluxes are much lower than the $p$ and He fluxes. Nonetheless, in the specific case of our best-fit propagation scenario (see below), we verified explicitly that including nuclei with $Z>2$ in the calculation changes the spectra of $\mathrm{He}$ (i.e., ${ }^{3} \mathrm{He}+{ }^{4} \mathrm{He}$ ) only by few percent and protons (i.e., $p+{ }^{2} \mathrm{H}$ ) by less than $1 \%$. This is also confirmed by the study in Ref. [32], where it is also shown that the $Z>2$ nuclei contribution to $\mathrm{He}$ is a few percent (although the contribution to ${ }^{2} \mathrm{H},{ }^{3} \mathrm{He}$ can be, instead, up to $20 \%-30 \%$ ).

\footnotetext{
${ }^{2}$ http://galprop.stanford.edu/.

${ }^{3}$ https://sourceforge.net/projects/galprop/.
} 


\section{METHODS AND DATA}

\section{A. Data}

As described in the Introduction, the main focus of the analysis is on the new AMS-02 measurements. We will thus use the published proton [2] and helium [3] AMS-02 spectra, and the available preliminary measurements of the antiproton over proton ratio [14]. The AMS-02 $p$ and He data extend up to a few TV. We thus complement them with $p$ and He CREAM measurements starting from a few TV up to $\sim 100$ TV. Finally, we use recently measured $p$ and He VOYAGER data [15] at low rigidities $\lesssim 1 \mathrm{GV}$, which are believed to be the first direct measurement of the local interstellar (LIS) flux, as a consequence of the fact that the probe crossed the solar Helio pause, leaving the solar system and entering in the interstellar space. A summary of the data sets used is presented in Table I. We use all data in rigidity, since this is the directly measured quantity by AMS-02 as opposed to the kinetic energy.

\section{B. Solar modulation}

To compare the AMS-02 fluxes to the GALPROP model predictions solar effects have to be taken into account. CRs are deflected and decelerated in the solar winds, whose activity varies in a $22 \mathrm{yr}$ cycle. The effect of this solar modulation [34] can be described phenomenologically by the force-field approximation [35,36], which is equivalent to taking into account only the adiabatic energy losses of the CRs propagating in the expanding solar wind. The process can be described by a single parameter $\phi$, the solar modulation, or Fisk potential [37], which links the total energy of the particles in the local interstellar space $E_{\mathrm{LIS}}$ to the energy $E$ observed in the detector at the Earth. The energy-differential flux $\Phi_{E}$ is then modulated by

$$
\begin{gathered}
E=E_{\mathrm{LIS}}-|Z| e \phi, \\
\Phi_{E}(E)=\frac{E^{2}-m^{2}}{E_{\mathrm{LIS}}^{2}-m^{2}} \Phi_{E, \mathrm{LIS}}\left(E_{\mathrm{LIS}}\right),
\end{gathered}
$$

where $Z$ is the charge number, $e$ the elementary charge, and $m$ the mass. The modulation potential can be approximately

TABLE I. Summary of the data sets used in this analysis.

\begin{tabular}{llll}
\hline \hline Experiment & Species & Rigidity range [GV] & Ref. \\
\hline AMS-02 & Proton & $1.0 \times 10^{0}-1.8 \times 10^{3}$ & {$[2]$} \\
AMS-02 & Helium & $1.9 \times 10^{0}-3.0 \times 10^{3}$ & {$[3]$} \\
AMS-02 & Antiproton ratio & $1.0 \times 10^{0}-0.2 \times 10^{3}$ & {$[14]$} \\
CREAM & Proton & $3.2 \times 10^{3}-2.0 \times 10^{5}$ & {$[33]$} \\
CREAM & Helium & $1.6 \times 10^{3}-1.0 \times 10^{5}$ & {$[33]$} \\
VOYAGER & Proton & $0.7 \times 10^{0}-1.0 \times 10^{0}$ & {$[15]$} \\
VOYAGER & Helium & $0.6 \times 10^{0}-2.3 \times 10^{0}$ & {$[15]$} \\
\hline \hline
\end{tabular}

derived from measurements of the neutron flux at Earth by various neutron monitor stations, since a strong anticorrelation is observed between the neutron flux and the solar activity [35,38]. Nonetheless, the procedure is affected by large uncertainties. In previous works, the usual procedure was to use this value, associate with it a "reasonable" uncertainty, and use it as a prior in the fit to CR data. Here, instead, we will use a novel procedure, similar to the one implemented in [39]. Assuming the measured VOYAGER $p$ and He fluxes, indeed, sample the LIS fluxes, we fit them with the unmodulated spectra, while, at the same time, the modulated spectra are fitted to the AMS-02 data. CREAM data, instead, are at very high energies where solar effects can be neglected. The explicitly used $\chi^{2}$ is reported in the next section in Eq. (10). We thus do not assume any specific prior for $\phi$ (in practice allowing a very large range; see Table II), and we let the VOYAGER data constrain it. In the future, monthly or weekly $p, \mathrm{He}$, and $\bar{p}$ data from AMS-02 should further help in better constraining $\phi$.

The force-field approximation, nonetheless, gives only a first-order description of the solar modulation process. A more complete description relies on a transport equation analogous to Eq. (1) but including the specific processes experienced by CRs while propagating in the solar magnetosphere [40]. The implementation of these models is, however, beyond the scope of the present work. Indeed, dedicated analyses, using time dependent proton flux of PAMELA [41,42] and VOYAGER data, suggest a strong rigidity and charge sign dependency of $\phi$ below a rigidity 5 GV $[39,43]$, indicating, in other words, a breakdown of the force-field approximation. Therefore, in the present analysis we use a fiducial lower rigidity threshold of $5 \mathrm{GV}$, although, we will also compare our fiducial fit results with those obtained including data down to $1 \mathrm{GV}$.

TABLE II. List of MultiNest and linear parameters in the fit and respective ranges of variation. See the text for a detailed description of the parameters.

\begin{tabular}{ll}
\hline \hline MultiNeSt parameters & Ranges \\
\hline$\gamma_{1}$ & $1.2-2.3$ \\
$\gamma_{2}$ & $2.0-2.9$ \\
$\gamma_{1, p}$ & $1.2-2.3$ \\
$\gamma_{2, p} \quad[\mathrm{GV}]$ & $2.0-2.9$ \\
$R_{0} \quad\left[\begin{array}{l}0 \\
s\end{array}\right.$ & $1.0-50$ \\
$\delta$ & $0.05-1.0$ \\
$D_{0} \quad\left[10^{28} \mathrm{~cm}^{2} / \mathrm{s}\right]$ & $0.1-0.9$ \\
$v_{\mathrm{A}} \quad[\mathrm{km} / \mathrm{s}]$ & $0.5-10.0$ \\
$v_{0, \mathrm{c}} \quad[\mathrm{km} / \mathrm{s}]$ & $0-60$ \\
$z_{h} \quad[\mathrm{kpc}]$ & $0-100$ \\
Linear parameters & $2-7$ \\
$A_{\mathrm{p}}$ & Ranges \\
$A_{\mathrm{He}}$ & $0.1-5.0$ \\
$\phi_{\text {AMS }} \quad[\mathrm{GV}]$ & $0.1-5.0$ \\
\hline \hline
\end{tabular}


As a final comment, we note that while Eq. (8) is nonlinear, only a single average potential $\phi$ is used in it. On the other hand, $\phi$ typically undergoes significant variations during the entire period over which the final averaged measured spectrum is provided (see [44] for a recent study of the time variation of $\phi$ ). We thus tested a fictitious case in which $\phi$ varies linearly in time from a value of $300 \mathrm{MV}$ to $700 \mathrm{MV}$ during a period of $2 \mathrm{yr}$, and we applied to a given model LIS spectrum the force-field approximation in small time bins, using in each bin the appropriate potential and then averaging at the end to derive the final modulated spectrum. This was compared with the flux obtained by direct application of Eq. (8) to the LIS using the average $\phi$. We found no appreciable difference between the two, indicating that Eq. (8) behaves linearly to a very good approximation. A posteriori, this can be justified in terms of the smallness of the $\phi$ parameter with respect to the larger rigidities involved.

\section{Fit procedure}

To scan the large parameter space we use the MuLTiNEST package [45]. MulTiNeST implements the algorithm of ellipsoidal nested sampling [46], allowing efficient likelihood evaluation and evidence calculation. As likelihood we use $\mathcal{L}=\exp \left(-\chi^{2} / 2\right)$ with

$$
\begin{aligned}
\chi^{2}= & \sum_{i} \frac{\left(\Phi_{\mathrm{AMS}, i}-\Phi_{M}\left(R_{i}\right)\right)^{2}}{\sigma_{\mathrm{AMS}, i}^{2}} \\
& +\sum_{D=V, C} \sum_{i} \frac{\left(\Phi_{D, i}-\Phi_{M, \mathrm{LIS}}\left(R_{i}\right)\right)^{2}}{\sigma_{D, i}^{2}},
\end{aligned}
$$

where $D=V, C$ indicates the VOYAGER and CREAM data sets with data points $i$ at rigidity and flux $\left(R_{i}, \Phi_{D, i}\right)$, with measured flux uncertainties flux $\sigma_{D, i}$, and $\Phi_{M \text {,LIS }}$ is the unmodulated model differential flux. Analogously, $\Phi_{\mathrm{AMS}, i}$ and $\sigma_{\mathrm{AMS}, i}$ indicate the AMS-02 data points and uncertainties, and $\Phi_{M}$ is the model flux modulated with a potential appropriate for AMS-02, $\phi_{\text {AMS }}$. We note here that reported fluxes by AMS-02 are actually the sum over the isotopes (protons and deuterons, and ${ }^{3} \mathrm{He}$ and ${ }^{4} \mathrm{He}$ ). The model spectra used in the $\chi^{2}$ are thus also summed over the isotopes for consistency. In the above $\chi^{2}$ we make the simplifying assumption that all the data points are uncorrelated. This is unlikely, since in most of the rigidity range the AMS-02 errors are dominated by systematic uncertainties rather than statistical ones, and systematic uncertainties are correlated in energy in various ways. A more rigorous treatment would require a deeper knowledge of the various systematic uncertainties and a way to model them, which, however, requires a detector-level analysis not available to us. We, nonetheless, note that neglecting the correlations should imply larger errors in the CR parameters estimated from the fit, and it should thus correspond typically to a conservative assumption.

The scan is intrinsically Bayesian, in the sense that MultiNest explores the posterior, which is specified by the likelihood $\mathcal{L}$ and the priors of the fitted parameters. Nonetheless, if the posterior and its tails have been sampled accurately enough, the likelihood samples collected by MultiNest can also be used for a frequentist analysis. This typically requires more aggressive MULTINEST settings for a more accurate sampling of the likelihood. We will use as a default the frequentist interpretation of the scan in terms of the profile likelihood [47], providing a comparison with the Bayesian interpretation in a specific case. As default, each scan is performed using for the MulTiNest settings 400 live points, an enlargement factor ef $r=0.6$ and a tolerance tol $=0.1$. We verified that the results are stable varying these settings. For each of the fits that we will describe in the following, the typical number of likelihood evaluations performed by MULTINEST is about 150,000. At the same time, with the settings described in Sec. II a GALPROP run requires about 45 CPU seconds, for a final total computational resources usage of about $\sim 3 \mathrm{CPU}$ months. The final efficiency (number of accepted steps over computed ones) of a typical scan is found to be $\sim 7 \%$. In the following, contour plots for two-dimensional profile likelihoods will be shown at the 1,2 , and $3 \sigma$ confidence levels calculated from a two-dimensional $\chi^{2}$ distribution. The error in the single parameters will be calculated from the related one-dimensional profile likelihoood and will be quoted at the $1 \sigma$ confidence level, i.e., from the condition $\Delta \chi^{2}=1$ with respect to the minimum $\chi^{2}$. We will also show 1 and $2 \sigma$ error bands around the best-fit spectra. They are derived from the envelope spectra of all the models lying within the 1 and $2 \sigma$ best-fit region in the full multidimensional parameter space.

In total we perform fits with up to 11 parameters, which can be grouped in two categories. The first one includes the parameters of the shape of the injection spectrum: $\gamma_{1, p}$, $\gamma_{2, p}, \gamma_{1}, \gamma_{2}, R_{0}$, and $s$. They denote the spectral indices, respectively, for protons and for the heavier species below and above the break at $R_{0}$ with smoothing $s$ [cf. Eq. (5)]. As shown in the next section, the freedom in the individual spectral indices for protons, denoted with the subscript $p$, is necessary to achieve a good description of the measured data. We provide a dedicated study investigating the limits of a possibly universal injection spectrum at the beginning of the next Sec. IV. The second category includes the parameters constraining the propagation, namely, the normalization $D_{0}$ and the slope $\delta$ of the diffusion coefficient, the Alfven velocity $v_{\mathrm{A}}$ related to reacceleration, the convection velocity $v_{0, c}$, and the halo size $z_{h}$. The above parameters are nonlinear, and a new GALPROP run has to be performed for every new parameter set. On the other hand, the fit includes three more parameters that do not require a new GALPROP run (for fixed values of the previous 
nonlinear parameters), namely, $A_{\mathrm{p}}, A_{\mathrm{He}}$, and $\phi_{\mathrm{AMS}}$, i.e., the normalization of the proton and helium fluxes as well as the solar modulation potential of AMS-02. For short we will call these parameters the linear parameters, even though $\phi_{\text {AMS }}$ does not act exactly linearly. In principle, these parameters can be treated in the same way as the other, and this would give a 14-dimensional parameter space to explore. We can, however, exploit the fact that they do not require a GALPROP evaluation to simplify the problem. We, thus, do not include these parameters in the set of parameters scanned by MultiNest, but, instead, we marginalize them on the fly for each set of the other 11 MultiNEST parameters. More precisely, for each nonlinear parameter set sampled by MultiNest we search for the minimum $\chi^{2}$ over the linear parameters, and we use this value to calculate the MultiNest likelihood. In this way, we use MultiNest to effectively scan over the 11-dimensional space that would be obtained from the 14-dimensional one marginalizing over the three linear parameters.

Any deviation of the normalizations $A_{i}$ from 1 implies a preference for a change in the CR species abundance with respect to the input value. As the normalization and spectrum of secondaries are calculated from the primaries' input relative abundances, rather than the ones rescaled by $A_{i}$, the input abundances need to be adjusted if the best fit prefers values of $A_{i}$ significantly different from 1 . We thus adjust the input abundances iteratively for all fits including antiprotons, repeating the fit until the normalizations $A_{i}$ converge to 1 . In practice, since the initial abundances are already very close to the ones preferred by the fit, only 1 or 2 iterations are typically required for convergence. To this purpose, the output proton spectrum from GALPROP is normalized to a value of $4.4 \times 10^{-9} \mathrm{~cm}^{-2} \mathrm{~s}^{-1} \mathrm{sr}^{-1} \mathrm{MeV}^{-1}$ at a kinetic energy of $100 \mathrm{GeV}$, and the parameter $A_{\mathrm{p}}$ is thus relative to this value. The helium $\left({ }^{4} \mathrm{He}\right)$ spectrum is instead normalized to a final (i.e., found after the iterations) input abundance of $7.80 \times 10^{4}$ relative to a proton abundance of $1.06 \times 10^{6}$. The parameter $A_{\mathrm{He}}$ is thus relative to this normalization. This value was found to be appropriate (i.e., giving $A_{\mathrm{He}}$ compatible with 1 after the fit) for all the fits performed, except for the case uni-PHePbar (see next section) where we used an input normalization of $9.48 \times 10^{4}$.

Three further parameters, required to specify the CR model spectra, are kept fixed or varied as a function of the other parameters. Specifically, the smoothness transition parameter $s_{1}$ for the second break $R_{1}$ is kept fixed to a value of 0.05 , given the sharp transition in this case, as can be seen directly in the $p$ and He spectra. The break itself and index after the break are fixed to $R_{1}=450 \mathrm{GeV}$ and $\gamma_{3}=\gamma_{2}-0.14$, for both $p$ and He. The latter two parameters have been fixed with the following procedure: At large rigidities above $\sim 100 \mathrm{GV}$ the $\mathrm{CR}$ spectrum is approximately given by the injection spectrum steepened by $\delta$,
$\Phi_{M}(R) \sim q_{R}(R) \cdot R^{-\delta} \sim\left\{\begin{array}{ll}\left(R / R_{1}\right)^{-\gamma_{2}-\delta} & R<R_{1} \\ \left(R / R_{1}\right)^{-\gamma_{3}-\delta} & \text { else }\end{array}\right.$.

Therefore it is possible to fit a broken power law directly to the data in order to determine the break position $R_{1}$ and the amount of the break $\Delta \gamma=\gamma_{3}-\gamma_{2}$. We performed two separate fits to $p$ only and He only data, using AMS-02 and CREAM data together. We find that both fits give compatible results for the break position and amount of break, with the values reported above. The determination of $R_{1}$ and $\Delta \gamma$ is where CREAM data play the main role in the analysis. For the general fits performed in the following, the weight of CREAM data is quite low, since the error bars are very large compared to AMS-02. Indeed, we verified that excluding CREAM data from the fit did not significantly change the fit constraints. We also tested the case in which we exclude the CREAM data from the fit, and for consistency we use values of $R_{1}$ and $\Delta \gamma$ determined from AMS-02 only. In this case the same procedure described above gives $R_{1}=270 \mathrm{GV}$ and $\Delta \gamma=-0.1$. We find that the impact of these changes on the secondary antiproton spectrum is only at the level of $10 \%-20 \%$ of the error bars of the data points above $50 \mathrm{GV}$. The fit constraints, consequently, are also not significantly affected.

The fit parameters, linear and nonlinear, and their explored ranges are summarized in Table II.

\section{RESULTS}

The results are presented as follows. We first discuss in Sec. IV A the possibility of fitting the data with a universal injection spectrum. In Sec. IV B we then proceed to the main analysis where separate spectral indices for the proton injection spectrum are allowed. In Sec. IV C we discuss the impact of the uncertainties related to the antiproton production cross section. A comparison between the frequentist and Bayesian results is shown in Sec. IV D. Finally, in Secs. IV E and IV F we check the robustness of the analysis results with respect to the chosen rigidity fit range and to the inclusion or exclusion of convection in the fit.

\section{A. Universal injection spectrum}

One of the unexpected features revealed by the precise measurements of PAMELA and AMS-02 is a significant discrepancy of the proton and helium spectral indices above $\sim 30 \mathrm{GV}$, with $\Delta \gamma_{p, \mathrm{He}}=0.101 \pm 0.014$ (PAMELA [1]) and $\Delta \gamma_{p, \mathrm{He}}=0.077 \pm 0.007$ (AMS-02 [2,3]). From the theoretical point of view the reason for this difference is unclear, and various possibilities have been discussed [4,5,7,48-50]. Moreover, acceleration in the sources above $\sim 30 \mathrm{GV}$ is expected to be charge independent, and therefore the same universal injection index is expected for $p$ and $\mathrm{He}$, as well as for the other species. We thus first investigate the possibility to fit the data assuming a 
universal injection index, attributing the difference in the observed indices to propagation effects. More in detail, we perform the fit as described in Sec. III, but we force the injection spectrum of protons and helium to be equal, i.e., $\gamma_{1, p}=\gamma_{1}$ and $\gamma_{2, p}=\gamma_{2}$, thus reducing the parameter space from 11 to 9 dimensions.

The fit is performed with two different data sets: in one case using only protons and helium (fit labeled as uni-PHe), and in the second case using proton, helium, and the antiproton-to-proton ratio (fit labeled uni-PHePbar).
Results are shown in Fig. 1. In the (uni-PHe) case we obtain a good fit with a minimal $\chi^{2}$ of 53.1 for a number of degrees of freedom (NDF) of 124. It can be seen in Fig. 1(c) that the best fit residuals with respect to the proton data are very flat in the fitted rigidity range. A similar result is obtained for the helium spectrum (not shown). The difference in the index between $p$ and $\mathrm{He}$ is explained by a significant production of secondary protons that soften the observed total (primaries plus secondaries) proton spectrum by the required $\sim 0.1$ value, with respect to the helium
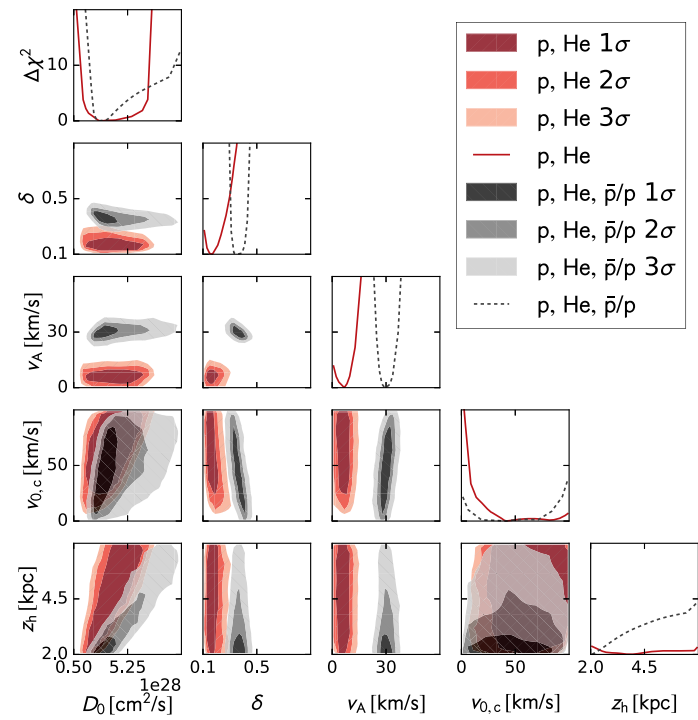

(a) Triangle plot for a selected set of propagation parameters.

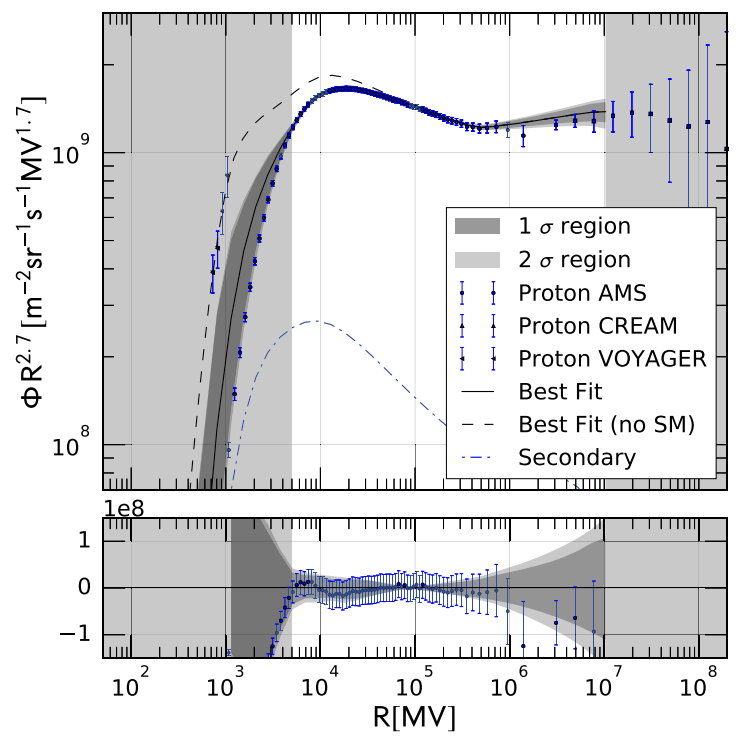

(c) Best fit results for protons for (uni-PHe).

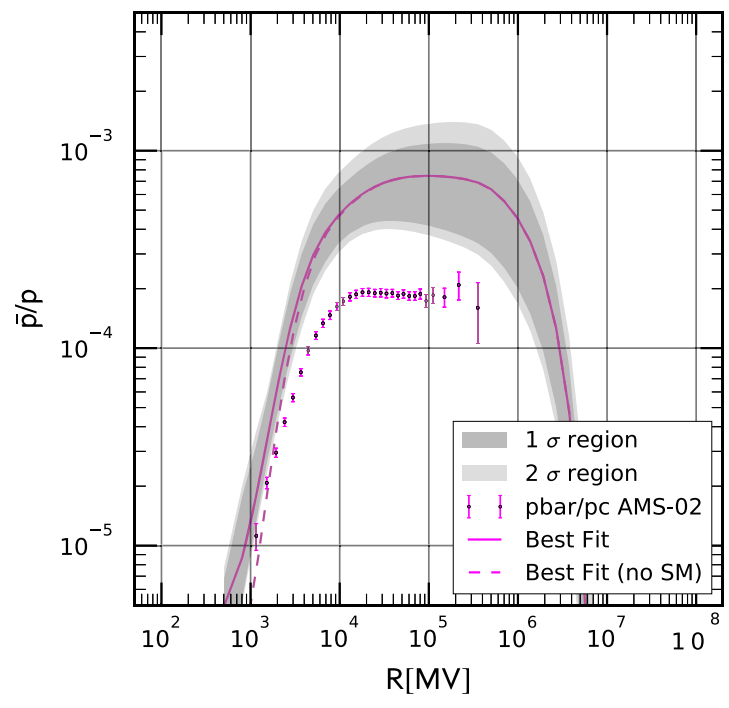

(b) Predicted antiproton spectrum for the (uni-PHe) fit.

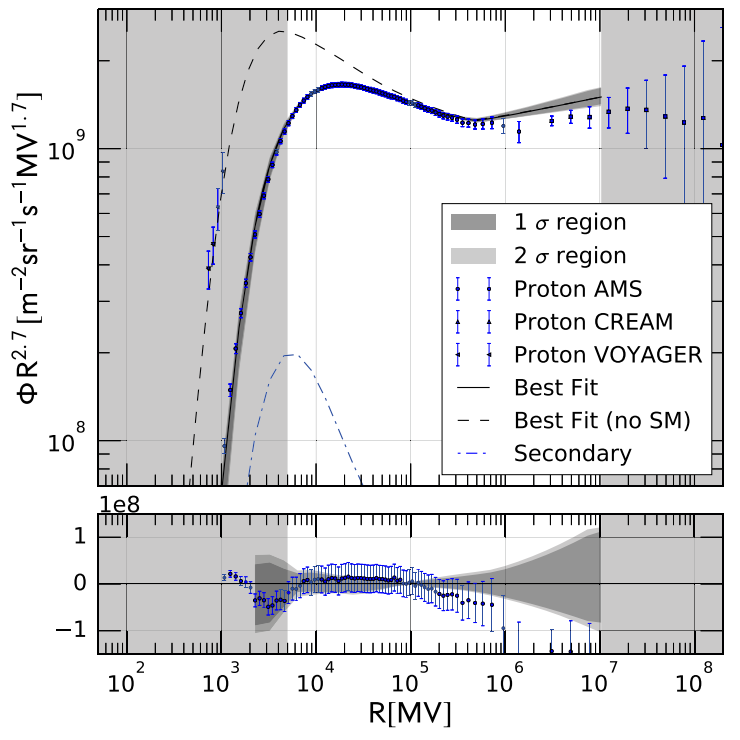

(d) Best fit results for protons for (uni-PHePbar).

FIG. 1. Comparison of the fit to data set (uni-PHe) and (uni-PHePbar) in the framework of a universal injection spectrum. In (c) and (d) the light-gray shaded regions indicate the rigidity range used in the fit. The lower panels in each of the plots show the residuals with respect to the best fit. 

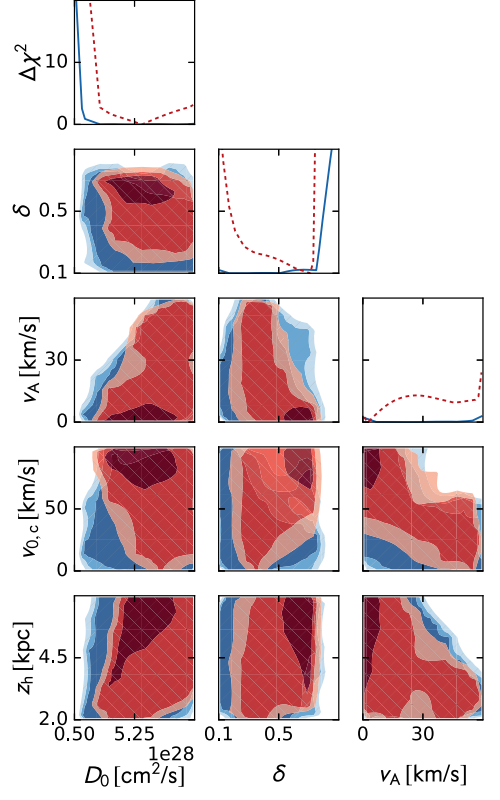

(a) (P) vs. (PHe)
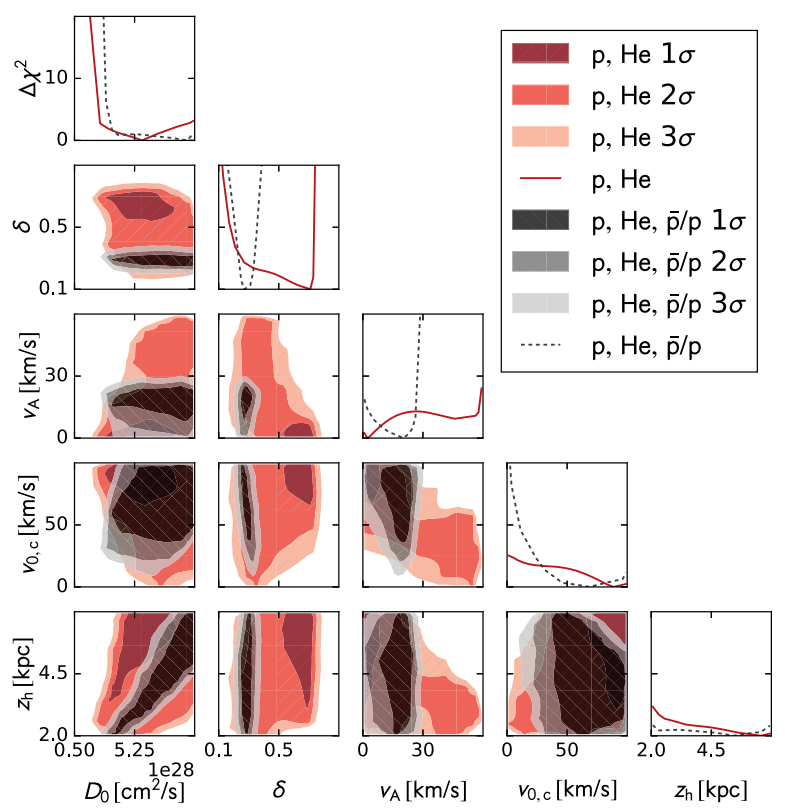

(b) (PHe) vs. (main)

FIG. 2. Comparison of fit results for the three data sets (P), (PHe), and (main) in the main fit framework (11 parameters) for a selected set of propagation parameters.

spectrum. In turn, this imposes strong constraints in the diffusion parameter space, as can be seen by the red contours in Fig. 1(a). In particular, a low value of $\delta \sim 0.15$ and a low amount of reacceleration $v_{\mathrm{A}} \sim 0$ are required. Although this scenario is appealing, it is ultimately revealed to be problematic. A first problem is the amount of solar modulation required by the fit, given by $\phi_{\text {AMS }}=300_{-75}^{+60} \mathrm{MV}$, which is quite low with respect to the neutron monitor expectation ${ }^{4}$ of $~ 500-600 \mathrm{MV}$ [38]. The second, more severe, problem is the fact that antiprotons are significantly overpredicted with respect to the observations, as shown in the lower panel of Fig. 1(b). This can also be seen from the result of the (uni-PHePbar) fit. In fact, the parameter space constraints from this fit, shown by the black-gray contours in Fig. 1(a), select a much larger value of $\delta \sim 0.4$ and of $v_{\mathrm{A}} \sim 30 \mathrm{~km} / \mathrm{s}$, which are incompatible, at high significance with the (uni-PHe) results. With the higher $\delta$ antiprotons data are now correctly produced but the amount of secondary protons is not enough anymore to explain the proton-helium index difference. This can clearly be seen from the systematic behavior of the residuals in Fig. 1(d), despite the fact that, formally, the fit is still reasonable, with a $\chi^{2} / \mathrm{NDF}$ of $140.4 / 147$. The (uni-PHePbar) fit also provides a more reasonable amount of solar modulation, with $\phi_{\text {AMS }}=780_{-40}^{+80} \mathrm{MV}$. These results are in qualitative agreement with [4,7], where $\mathrm{He}$ spallation effects were studied to explain the difference in

\footnotetext{
${ }^{4}$ An updated table for the solar modulation potential up to 2016 is available at http://cosmicrays.oulu.fi/phi/Phi_mon.txt.
}

slope between proton and He, and similar difficulties in explaining secondaries' spectra were encountered. Note also that, as explained in Sec. II, we are neglecting the eventual contribution of secondary protons from $Z>2$ nuclei. This is not expected to be crucial, since, even in the case this contribution would be large (20\%-30\%), this would not solve the above issues.

In the light of the above results, we will adopt in the following as the main scenario the one in which the proton and helium spectral indices are varied independently.

\section{B. Main fit}

Using the 11-dimensional setup discussed in Sec. III, we will now perform fits to different data sets to test the selfconsistency of the results. In particular, we consider the following 3 fits: using only proton data $(\mathrm{P})$, proton and helium data (PHe), and proton, helium, and antiproton data (main). Figure 2 shows how the propagation parameter space successively shrinks by going from data set $(\mathrm{P})$ to (main). As expected, because of the large degeneracy of the parameters in case $(\mathrm{P})$ nearly the whole sampled parameter space is allowed. Adding helium data results in a tendency against reacceleration, a preference toward large values for the convection velocity $v_{c, 0} \gtrsim 50 \mathrm{~km} / \mathrm{s}$, and a diffusive halo height $z_{h} \gtrsim 4 \mathrm{kpc}$. The constraints, however, are not extremely strong, and at the $\sim 3 \sigma$ level again almost the whole parameter space is allowed.

Figure 2(b) shows the comparison between (PHe) and (main) results. As expected the secondary antiprotons give tight constraints on the rigidity dependence of diffusion 
$\delta \sim 0.3$, while the usual degeneracy in $D_{0}-z_{h}$ appears, and no constraints on $z_{h}$ can be inferred. This is also expected since strong constraints on $z_{h}$ can be achieved only using precise data on radioactive clocks like ${ }^{10} \mathrm{Be} /{ }^{9} \mathrm{Be}$, which are not yet available. Finally, $v_{\mathrm{A}}$ and $v_{0, c}$ are poorly constrained individually apart from a tendency to not prefer strong reacceleration $v_{\mathrm{A}} \lesssim 30 \mathrm{~km} / \mathrm{s}$ and a favor for large convection $v_{0, c} \gtrsim 50 \mathrm{~km} / \mathrm{s}$. This is mainly due to the fact that they have approximately degenerate effects on the spectra, and only a combination of the two parameter is well constrained. Interestingly, a fit with only convection and no reacceleration seems, thus, possible. Indeed, some critical view on reacceleration has recently been discussed [51]. It can be seen that (PHe) and (main) are not compatible at the $2 \sigma$ level, although they became fully compatible at the $3 \sigma$ level. Given the very small error bars of AMS-02 it is perhaps expected that incompatibilities at the $2 \sigma$ level might appear, due to the fact that the level of complexity of the fitted models is likely starting to be not comparably adequate. We thus deem the compatibility at $3 \sigma$ reasonable.

For the case (main) the complete parameter space is shown in Fig. 3, and the likelihood profiles of the linear parameters are given in Fig. 4. It can be seen that indeed $A_{\mathrm{He}}$ is compatible with 1 at $1 \sigma . A_{\mathrm{p}}$ does actually differ from 1 , but only at the $3 \%$ level which is much smaller than the uncertainties in the other parameters. We thus did not perform a further fit iteration, readjusting again the input proton normalization. The minimal $\chi^{2} / \mathrm{NDF}$ of the best fit point is $39.0 / 145$. The agreement between data and model is thus very good (cf. also Figs. 5 and 6). There are no systematics features in the residuals of proton and helium spectra. The small residual structures in the antiproton-toproton ratio are within the error band. The best-fit

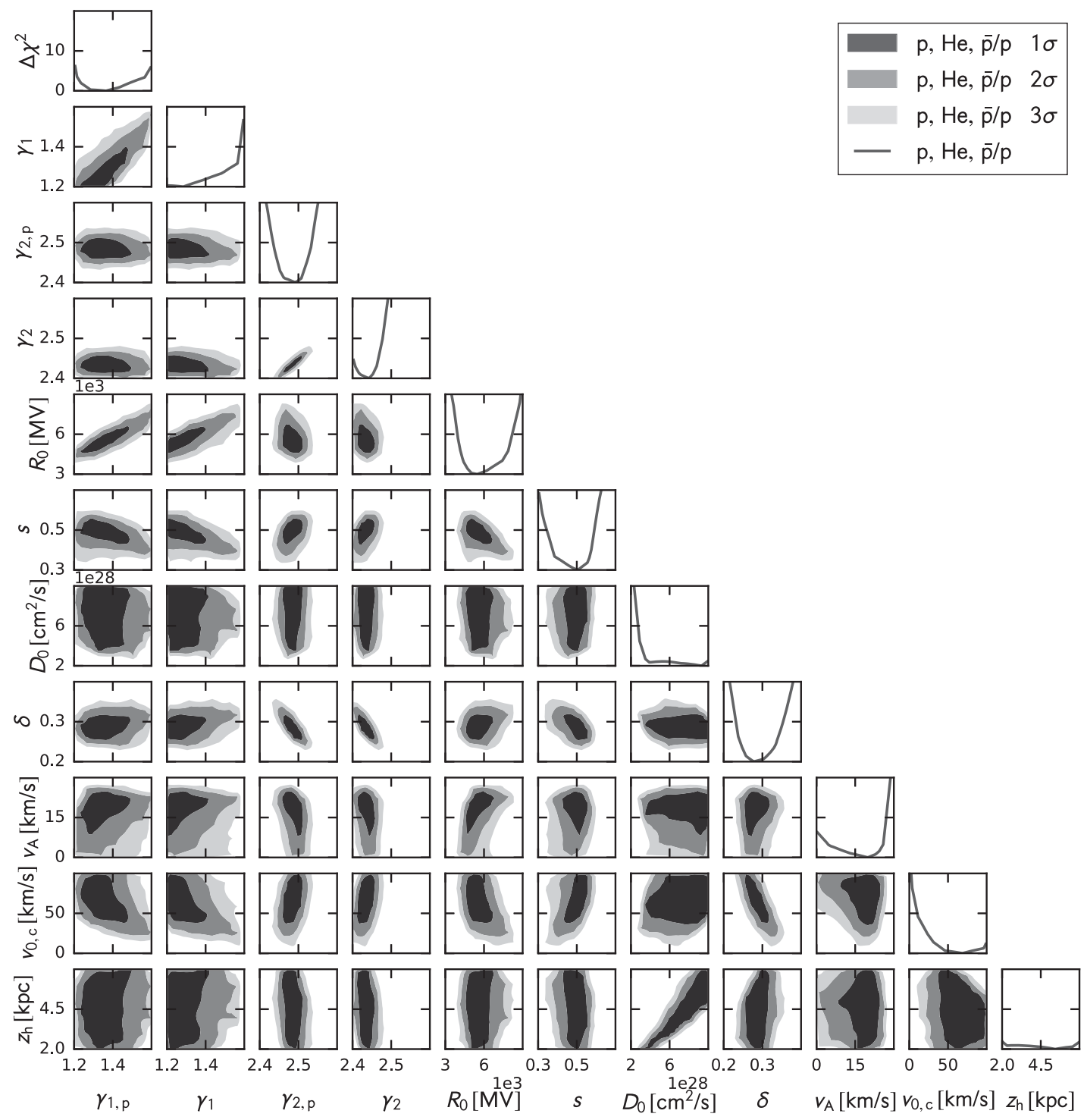

FIG. 3. Full triangle plot for the results of the main fit using protons, helium, and antiprotons (main). 

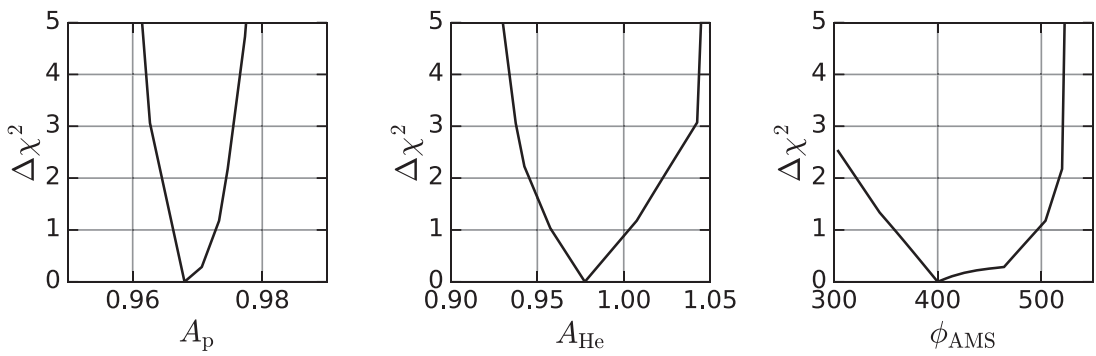

FIG. 4. Profiles of the linear parameters for the (main) fit.

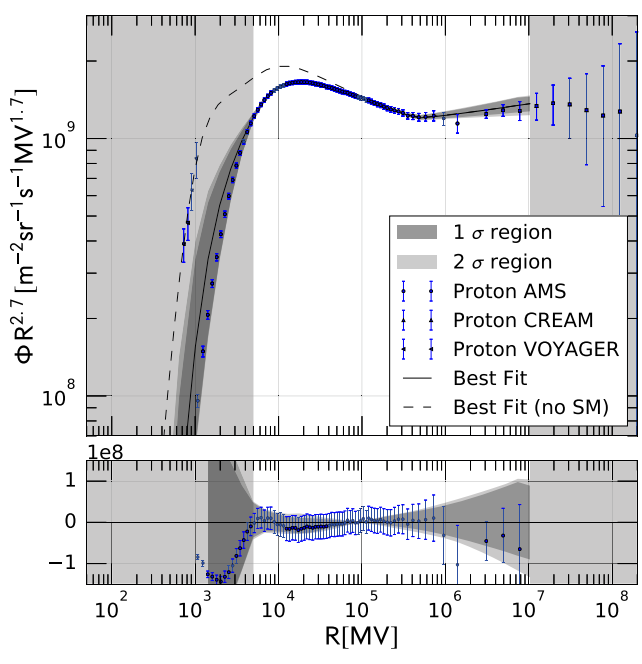

(a) Proton

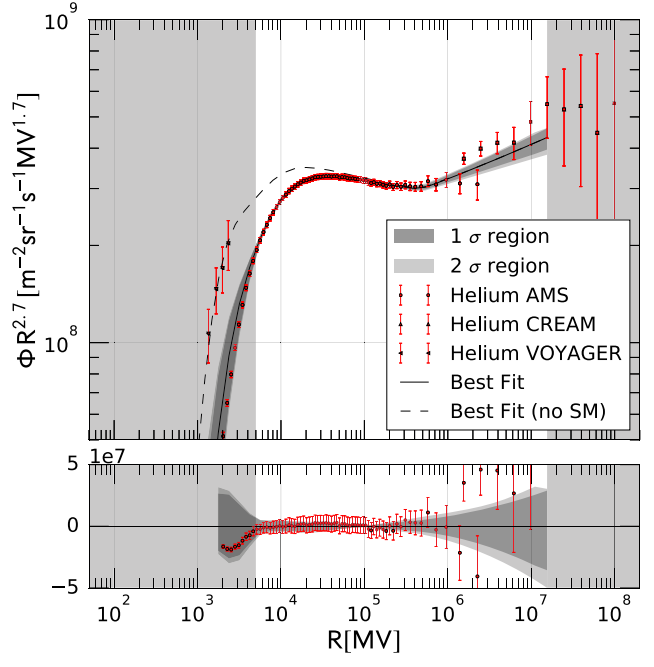

(b) Helium

FIG. 5. Comparison between data and best-fit model for the main fit framework (main): 11 parameters and fit to proton, helium, and antiprotons.

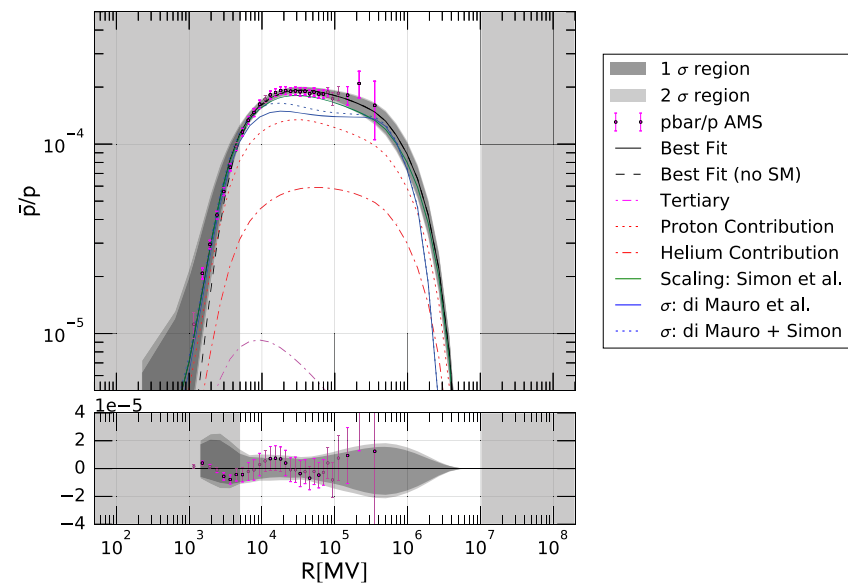

FIG. 6. Comparison between $\bar{p} / p$ data and best-fit model for the main fit (main) framework (11 parameters and fit to proton, helium, and antiprotons). The various contributions to the total antiproton spectrum are also shown, as well as different production cross section models. The proton contribution includes only $p p$ production, whereas the helium contribution also includes $p \mathrm{He}$ and $\mathrm{He} p$ production. parameter values and their uncertainties are summarized in Table III. Finally, also the VOYAGER $p$ and He measurements are well fitted by the unmodulated model spectra, as shown in Fig. 5. The best fit for the position of the break $R_{0}$ is compatible with $5 \mathrm{GV}$, our lower rigidity threshold, indicating that a low rigidity break is not necessary to fit the data. We will comment more on this point in Sec. IV E where we show the results of the fit including data down to $1 \mathrm{GV}$.

The effect on the fit of the parameter $s$ introduced in this work can be inferred from Fig. 3. It can be seen that, apart from the expected degeneracy with the break position, $s$ has only mild degeneracies with the other parameters. Indeed, performing explicitly a fit without $s$ (a sharp break), we found that only the ranges for $v_{\mathrm{A}}$ and $v_{0, c}$ slightly change, the two parameters being in any case not well determined. The main effect of $s$ is, instead, to provide an overall better fit to the data and flatter residuals. It is unclear if the need for $s$ in the fit implies, indeed, that the injection spectra have a smooth break, or, alternatively, if $s$ is compensating for a different effect, as, e.g., systematics in the modeling of the solar modulation. 
TABLE III. Best fit values and $1 \sigma$ errors for the various fits. If the error coincides with the upper or lower bound of the prior range, the error value is given in italic.

\begin{tabular}{|c|c|c|c|c|c|c|c|c|c|c|}
\hline \multicolumn{2}{|c|}{ Fit parameters } & (uni-PHe) & (uni-PHePbar) & $(\mathrm{P})$ & $(\mathrm{PHe})$ & (main) & (di Mauro) & $(1 \mathrm{GV})$ & \multicolumn{2}{|c|}{$($ noVc-1 GV) (noVc-5 GV) } \\
\hline$\gamma_{1, p}$ & & & $\cdots$ & $1.52_{-0.32}^{+0.21}$ & $1.27_{-0.07}^{+0.11}$ & $1.36_{-0.10}^{+0.07}$ & $1.38_{-0.10}^{+0.07}$ & $1.32_{-0.12}^{+0.05}$ & $1.61_{-0.10}^{+0.06}$ & $1.76_{-0.04}^{+0.07}$ \\
\hline$\gamma_{2, p}$ & & $\cdots$ & $\cdots$ & $2.52_{-0.45}^{+0.12}$ & $2.069_{-0.069}^{+0.098}$ & $2.493_{-0.026}^{+0.010}$ & $2.499_{-0.014}^{+0.026}$ & $2.455_{-0.007}^{+0.014}$ & $2.421_{-0.014}^{+0.010}$ & $2.454_{-0.014}^{+0.026}$ \\
\hline$\gamma_{1}$ & & $1.92_{-0.14}^{+0.08}$ & $1.50_{-0.12}^{+0.07}$ & $\ldots$ & $1.53_{-0.11}^{+0.24}$ & $1.29_{-0.09}^{+0.04}$ & $1.26_{-0.06}^{+0.10}$ & $1.32_{-0.12}^{+0.06}$ & $1.65_{-0.11}^{+0.07}$ & $1.70_{-0.07}^{+0.06}$ \\
\hline$\gamma_{2}$ & & $2.582_{-0.034}^{+0.010}$ & $2.404_{-0.022}^{+0.006}$ & $\cdots$ & $2.003_{-0.003}^{+0.094}$ & $2.440_{-0.018}^{+0.006}$ & $2.451_{-0.010}^{+0.018}$ & $2.412_{-0.006}^{+0.012}$ & $2.381_{-0.010}^{+0.010}$ & $2.407_{-0.014}^{+0.022}$ \\
\hline$R_{0}$ & {$[\mathrm{GV}]$} & $8.16_{-1.54}^{+1.22}$ & $8.79_{-1.55}^{+1.17}$ & $4.38_{-1.54}^{+3.23}$ & $10.5_{-1.59}^{+1.40}$ & $5.54_{-0.54}^{+0.76}$ & $5.44_{-0.54}^{+0.54}$ & $5.52_{-0.83}^{+0.33}$ & $7.01_{-0.54}^{+0.98}$ & $8.63_{-0.76}^{+0.98}$ \\
\hline$s$ & & $0.32_{-0.02}^{+0.08}$ & $0.41_{-0.07}^{+0.09}$ & $0.48_{-0.31}^{+0.16}$ & $0.59_{-0.04}^{+0.16}$ & $0.50_{-0.04}^{+0.02}$ & $0.50_{-0.03}^{+0.05}$ & $0.43_{-0.03}^{+0.04}$ & $0.31_{-0.03}^{+0.03}$ & $0.32_{-0.05}^{+0.04}$ \\
\hline$\delta$ & & $0.16_{-0.02}^{+0.03}$ & $0.36_{-0.03}^{+0.04}$ & $0.29_{-0.18}^{+0.46}$ & $0.72_{-0.11}^{+0.01}$ & $0.28_{-0.01}^{+0.03}$ & $0.27_{-0.04}^{+0.02}$ & $0.32_{-0.02}^{+0.03}$ & $0.40_{-0.01}^{+0.01}$ & $0.36_{-0.02}^{+0.02}$ \\
\hline$D_{0}$ & {$\left[10^{28} \mathrm{~cm}^{2} / \mathrm{s}\right]$} & $2.77_{-0.53}^{+2.95}$ & $2.83_{-0.50}^{+0.90}$ & $4.78_{-3.49}^{+5.22}$ & $5.95_{-1.37}^{+0.83}$ & $9.30_{-5.48}^{+0.70}$ & $9.04_{-3.95}^{+0.96}$ & $8.19_{-4.68}^{+1.81}$ & $4.92_{-2.36}^{+1.12}$ & $4.60_{-2.04}^{+2.71}$ \\
\hline$v_{\mathrm{A}}$ & {$[\mathrm{km} / \mathrm{s}]$} & $6.80_{-2.73}^{+1.18}$ & $29.2_{-1.47}^{+2.80}$ & $21.2_{-21.2}^{+38.8}$ & $1.84_{-1.08}^{+2.36}$ & $20.2_{-6.33}^{+3.26}$ & $18.2_{-5.91}^{+3.15}$ & $25.0_{-2.30}^{+0.92}$ & $22.8_{-1.05}^{+1.46}$ & $20.7_{-3.43}^{+1.14}$ \\
\hline$v_{0, \mathrm{c}}$ & {$[\mathrm{km} / \mathrm{s}]$} & $40.9_{-5.89}^{+59.1}$ & $40.2_{-25.2}^{+38.1}$ & $5.82_{-5.82}^{+94.2}$ & $87.8_{-7.57}^{+12.2}$ & $69.7_{-24.7}^{+22.0}$ & $57.3_{-12.3}^{+41.1}$ & $44.0_{-16.5}^{+8.4}$ & $\cdots$ & . \\
\hline$z_{\mathrm{h}}$ & {$[\mathrm{kpc}]$} & $3.77_{-1.77}^{+3.23}$ & $2.04_{-0.04}^{+0.40}$ & $4.22_{-2.22}^{+2.78}$ & $6.55_{-1.63}^{+0.45}$ & $5.43_{-3.43}^{+1.57}$ & $5.84_{-3.84}^{+1.16}$ & $6.00_{-4.00}^{+1.00}$ & $5.05_{-3.05}^{+1.95}$ & $4.12_{-2.12}^{+2.88}$ \\
\hline$\phi_{\mathrm{AM}}$ & & $300_{-80}^{+60}$ & $780_{-40}^{+80}$ & $620_{-195}^{+180}$ & $580_{-115}^{+45}$ & $400_{-40}^{+90}$ & $360_{-45}^{+115}$ & $700_{-50}^{+20}$ & $640_{-20}^{+20}$ & $340_{-125}^{+45}$ \\
\hline
\end{tabular}

In the following sections we will take (main) as baseline for further cross-checks and systematic studies.

\section{Antiproton production cross section}

The lack of precise measurements of the antiproton production cross section [cf. Eq. (7)] constitutes an important systematic uncertainty in the interpretation of the precisely measured fluxes [16,52]. Detailed measurements for the antiproton production exist only for proton-proton inelastic scattering up to center of mass energies of $\sim 63 \mathrm{GeV}^{5}$ [16]. For larger energies or different target particles it is necessary to extrapolate and/or rescale the cross sections, leading to model dependent results. Furthermore, in the proton-proton inelastic scattering, only the antiproton production cross section is directly measured, while no measurement is available for the antineutron (which subsequently decay into antiproton) production cross section. In principle, from isospin symmetry the latter is expected to be equal to the former. On the other hand, a measurement from NA49 [53] suggests that the antineutron cross section is actually slightly larger than the antiproton one. Further details are discussed in Refs. [16,54]. In this section we compare our default choice of $p+p \rightarrow \bar{p}+X$ cross section given by Tan and $\mathrm{Ng}$ [55] as implemented in GALPROP with the more recent study in [16].

As mentioned above, in order to get the antiproton $(\bar{p})$ production cross section for arbitrary projectiles and targets, denoted by subscripts $P$ and $T$, respectively, we need a scaling from the $p p$ collision cross section.

\footnotetext{
${ }^{5}$ Some sparse measurement up to $\sim 200 \mathrm{GeV}$ also exists [16].
}

A common approximation is to treat the projectile as a flux of $A_{P}$ protons with momenta $p_{P} / A_{P}$. The target is instead scaled according to the semiclassical model where the volume scales as $A_{T}$ and the area as $A_{T}^{2 / 3}$. For the production of antineutrons $(\bar{n})$, which subsequently decay into antiprotons and contribute directly to the flux, we assume a scaling of 1.3. Comprehensively, we get

$$
\begin{aligned}
\frac{\mathrm{d} \sigma_{P, T}^{(\bar{p})}\left(p_{P}, p_{\bar{p}}\right)}{\mathrm{d} p_{\bar{p}}} & =A_{P} A_{T}^{2 / 3} \frac{\mathrm{d} \sigma_{p, p}^{(\bar{p})}\left(\frac{p_{P}}{A_{P}}, p_{\bar{p}}\right)}{\mathrm{d} p_{\bar{p}}} \\
\frac{\mathrm{d} \sigma_{P, T}^{(\bar{n})}\left(p_{P}, p_{\bar{n}}\right)}{\mathrm{d} p_{\bar{n}}} & =1.3 \frac{\mathrm{d} \sigma_{P, T}^{(\bar{p})}\left(p_{P}, p_{\bar{n}}\right)}{\mathrm{d} p_{\bar{n}}}
\end{aligned}
$$

The dominant contribution of the antiproton flux comes from $p p$ collisions (cf. Fig. 6). The contribution from nonproton projectiles and/or targets plays a subdominant role, since both the interstellar helium gas contribution and the $\mathrm{CR}$ abundance of helium amount to only roughly $10 \%$. The main uncertainty arising from the scaling is thus related to the antineutron cross section, since the antineutrons are produced directly in the $p p$ collisions. The scaling described above gives similar results to the one implemented as default in GALPROP. Figure 6 shows the comparison between the total antiproton flux for our best fit model in the main fit framework using the default cross section and the one derived in [16] [their Eq. (13)]. We can see that overall the cross section from [16] predicts a lower normalization of the antiproton flux by about $\sim 20 \%$. Also the shape is slightly different with a mild hardening of the flux starting at about $\sim 20 \mathrm{GV}$. In the plot, for completeness, we also show the contribution 


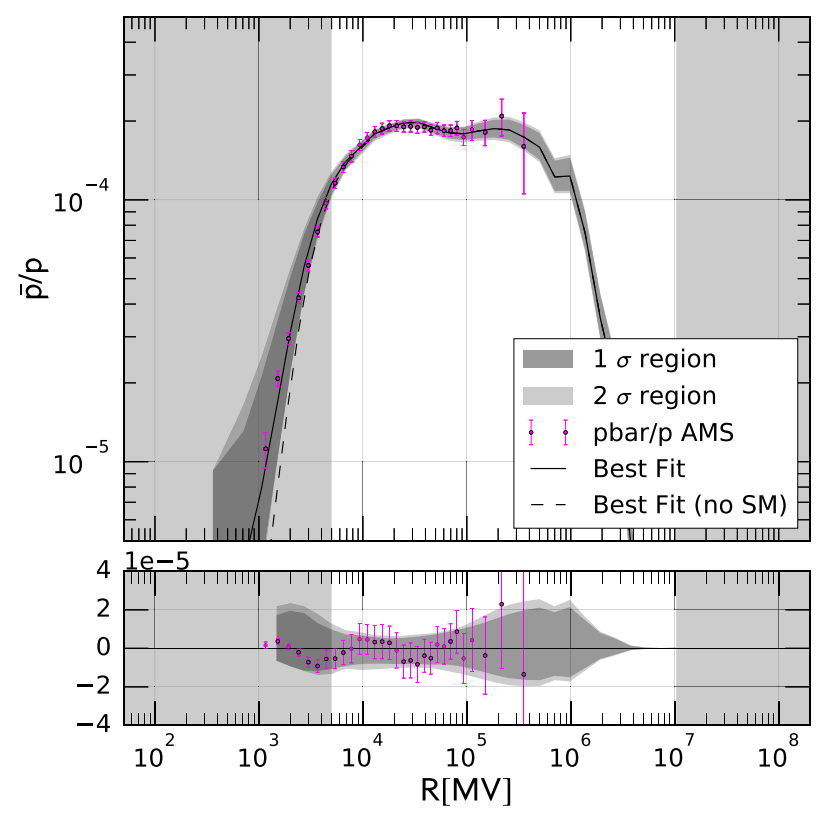

FIG. 7. $\quad \bar{p} / p$ ratio for fit using the antiproton production cross section from di Mauro et al. [16].

from tertiary antiprotons, as well as the separate contributions from proton collisions and collisions involving helium. Finally, we also tested a different scaling available in GALPROP due to Simon et al. [56] applied to the two cross sections, and we find in both cases that this introduces a $\sim 5 \%$ variation with respect to the flux with the default scaling.
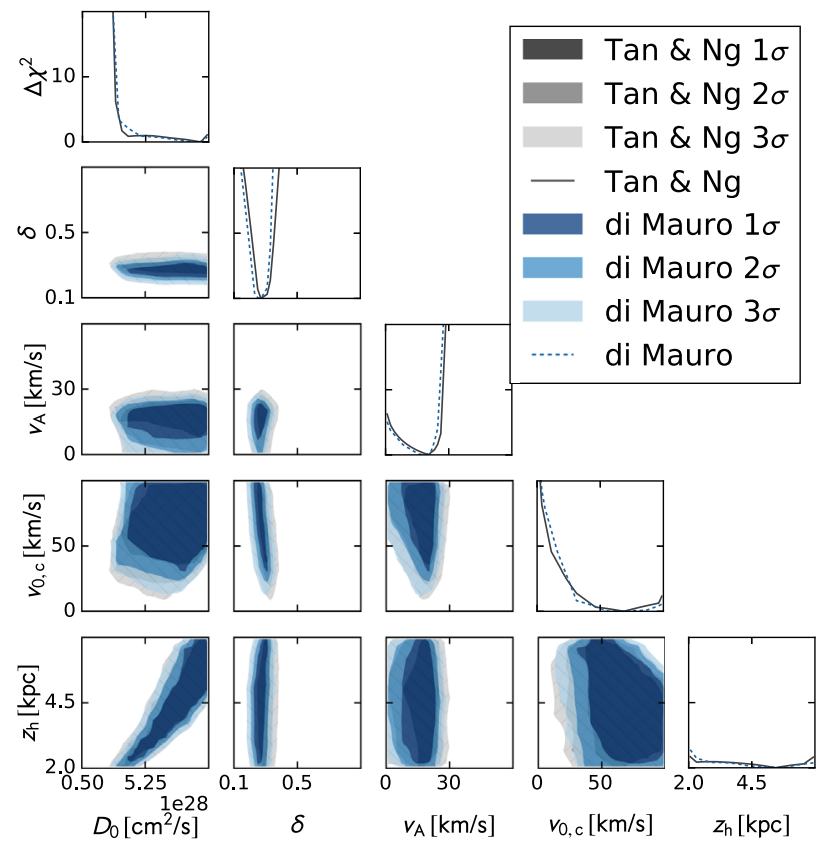

FIG. 8. Comparison of the baseline fit with the fit using the antiproton production cross section from di Mauro et al.
Intriguingly, as seen in Fig. 6, the flux derived from the cross section from [16] seems to slightly better fit the observed antiproton spectrum. We thus repeat the fit using the new cross section (di Mauro). Figure 7 shows the results using the parametrization from their Eq. (13). As expected the high-energy part fits better than in the (main) fit, but the shape of the low-energy tail does not exactly match the measurements, leaving a similar amount of systematics in the residuals. As expected the lower normalization of the cross section compared to GALPROP is compensated by a slightly lower value of $\delta$ which drops to 0.27 , which can be seen from Fig. 8. The other parameters are not changed. We also test the cross section from their Eq. (12), which leads to a similar result.

\section{Frequentist vs Bayesian interpretation}

The results of this analysis are interpreted in the frequentist approach, whereas previous analyses were mostly done in a Bayesian framework. We thus compare the two approaches for the main fit case. In the Bayesian case we derive constraints from the posterior distribution, as opposed to the frequentist case where we use only the likelihood function. The Bayesian posterior is interpreted as probability distribution once the priors in model parameters are specified. In our case, prior ranges are as specified in Table II, and they are linear in all the parameters. Twodimensional posteriors for two given parameters are derived marginalizing (i.e., integrating) the full posterior over the remaining parameters. In practice, marginalized posteriors are a natural output of the Monte Carlo based

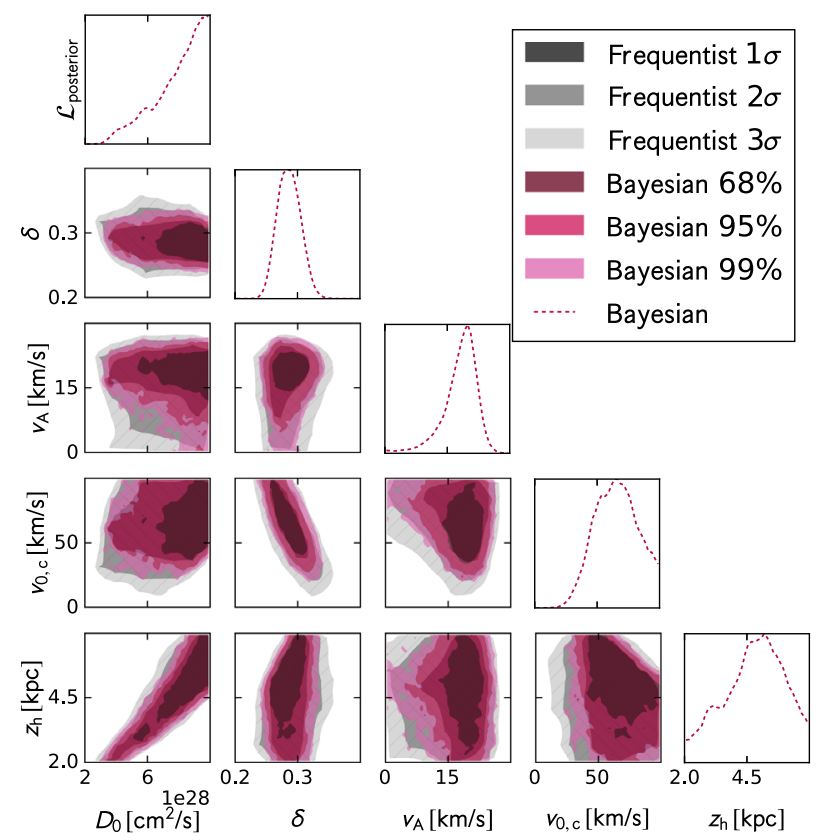

FIG. 9. Comparison of the baseline fit (main) with the Bayesian interpretation. 

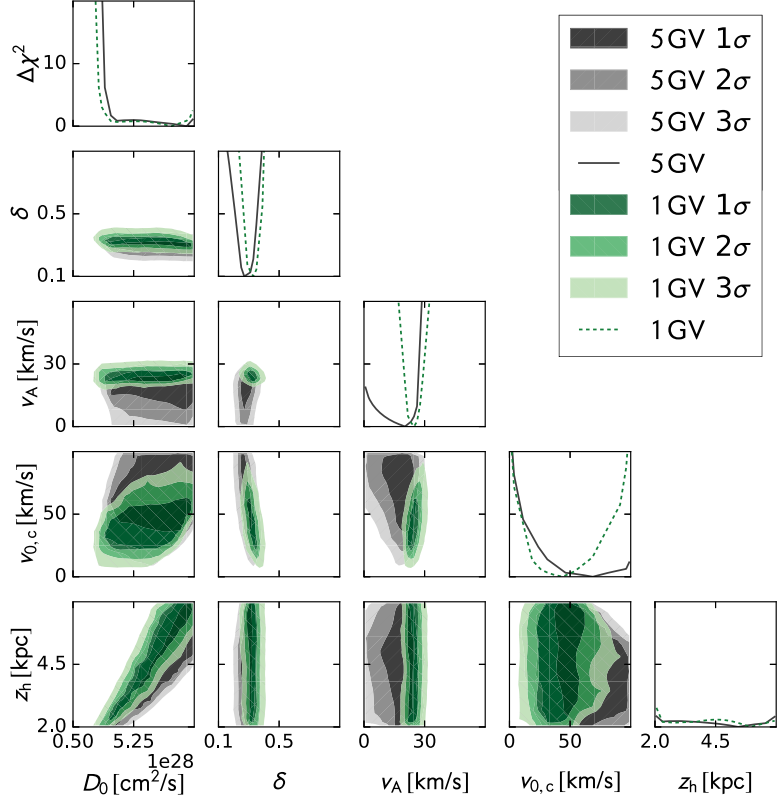

(a) Propagation parameters.

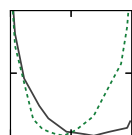

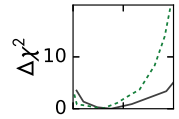
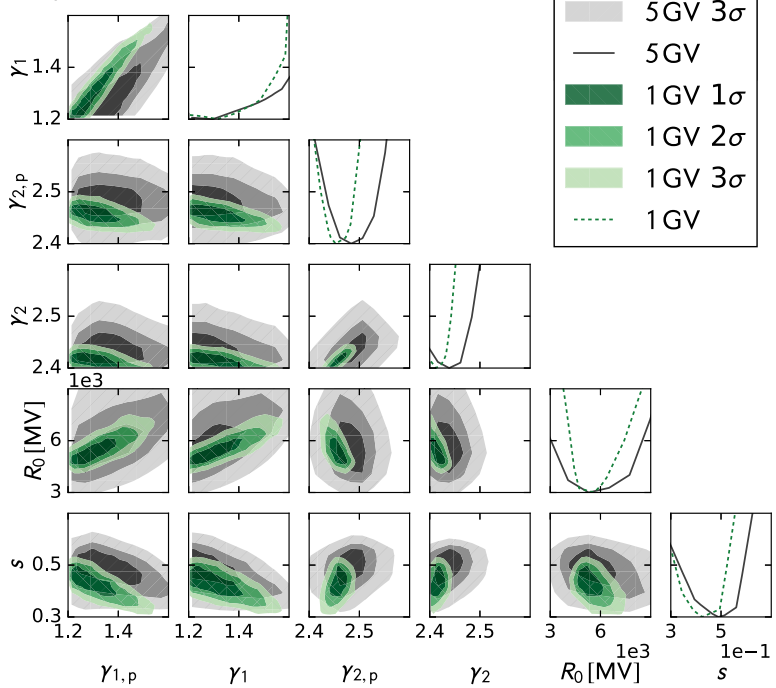

(b) Injection parameters

FIG. 10. Comparison of the baseline fit (main) to a fit including data down to $1 \mathrm{GV}$ (1 GV) for (a) propagation and (b) injection parameters.

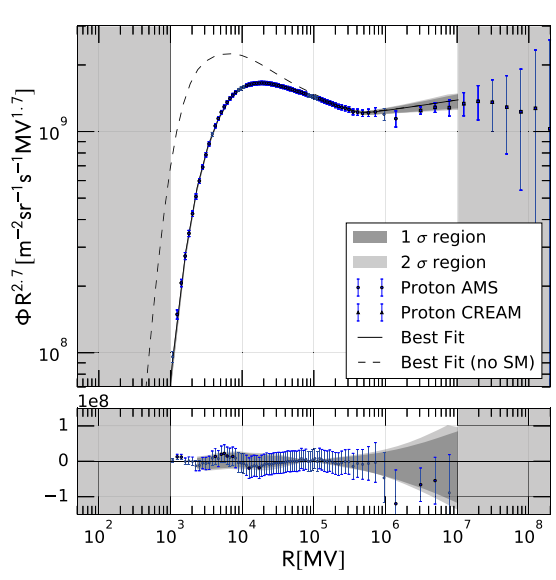

(a) Proton

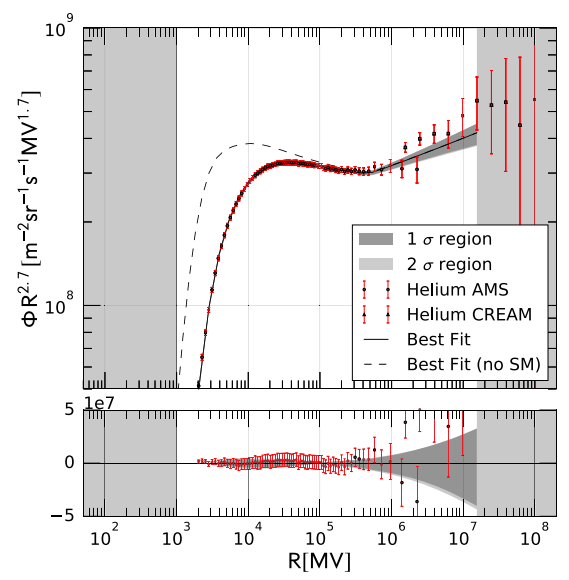

(b) Helium

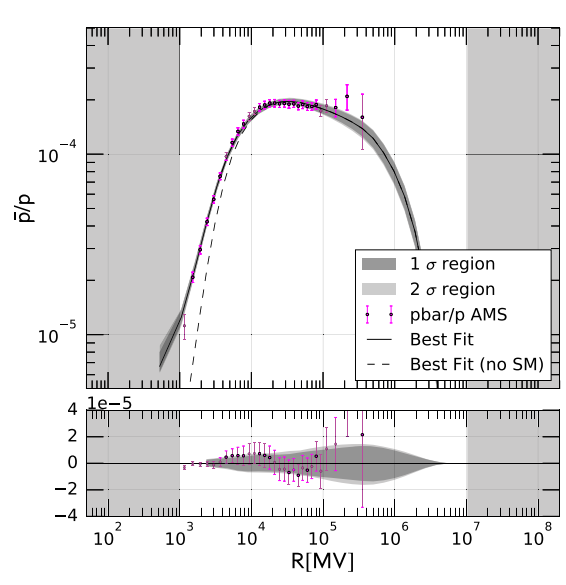

(c) $\bar{p} / p$

FIG. 11. Comparison between data and best-fit model for the global analysis including proton, helium, and antiprotons down to $1 \mathrm{GV}$ (1 GV). 
scanning technique, and integrals do not need to be performed explicitly. Bayesian contours are then derived integrating the marginalized posterior up to the specified confidence level. In Fig. 9 we show the triangle plot for a selected set of parameters and compare the $1 \sigma$ to $3 \sigma$ frequentist contours with the equivalent Bayesian contours. It can be seen that the two approaches give compatible results, with the frequentist case being slightly more conservative. In fact, the two approaches are expected to give compatible results in the limit in which the data are constraining enough, and the effect of the priors start to be subdominant. The above results indicate indeed that the results are data driven rather than prior driven, and thus robust.

\section{E. Fit down to $1 \mathrm{GV}$}

As explained in Sec. III we limit our fit range to $R>5 \mathrm{GV}$ to reduce the effects of the solar modulation. Thus we avoid the rigidity range indicating rigidity and charge sign dependence of the solar modulation potential. In this section we investigate the effect of extending the fit range down to $1 \mathrm{GV}$ [labeled $(1 \mathrm{GV})]$. The results of the fit are shown in Figs. 10 and 11. Figure 11 shows that a good fit is achieved with flat residuals all over the fitted energy range. The $\chi^{2} / \mathrm{NDF}$ has a value of $70.3 / 175$. From Fig. 10(a) it can be seen that the two fits give consistent results at the level of a bit more than $2 \sigma$. The slight shift of about 0.04 in the value of $\delta$ from $0.28_{-0.01}^{+0.03}$ to $0.32_{-0.02}^{+0.03}$ can be considered as an estimate of the systematic error on this parameter. Regarding reacceleration and convection, adding data down to $1 \mathrm{GV}$ contributes to basically break their degeneracy providing a strong constraint on $v_{\mathrm{A}}=$ $25.0_{-2.30}^{+0.92} \mathrm{~km} / \mathrm{s}$ and a lower value of $v_{0, c}=44.4_{-19.8}^{+12.2} \mathrm{~km} / \mathrm{s}$. At the same time tight constraints on the index below the break $\gamma_{1}=1.32_{-0.12}^{+0.06}, \gamma_{1, p}=1.32_{-0.12}^{+0.05}$ and on the
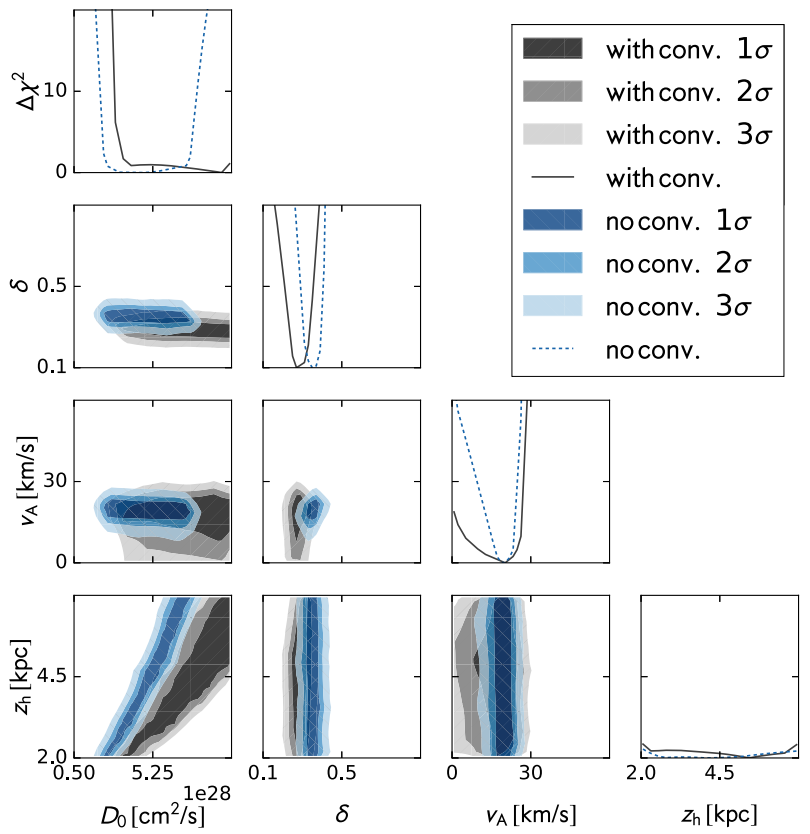

FIG. 12. Comparison of the propagation parameters from the baseline analysis (main) to a diffusion model without convection (noVc-5 GV).

break itself $R_{0}=5.52_{-0.83}^{+0.33} \mathrm{GV}$ appear. The latter value suspiciously coincides with the rigidity below which the constant solar modulation potential approximation should start to fail. For this reason it is unclear if the presence of the break is indeed physical or if it is a way for the fit to compensate for the nonprecise solar modulation modeling. At the same time it is equally unclear if the resulting values of $v_{\mathrm{A}}$ and $v_{0, c}$ are robust or are biased by the possibly incorrect solar modulation. As mentioned also in Sec. IV B, to settle the issue a more careful study of the solar modulation effect will be

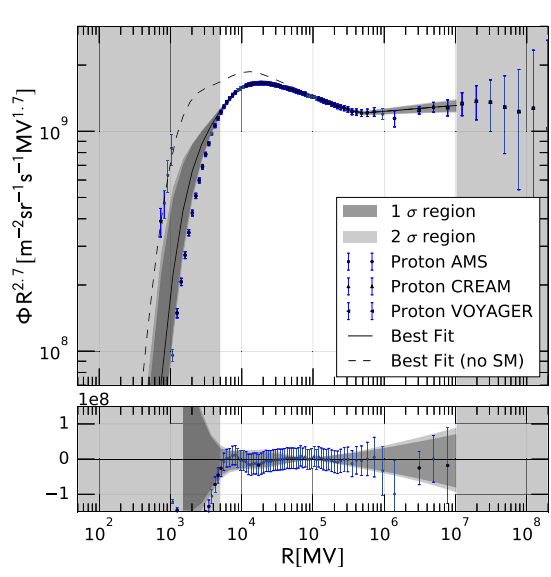

(a) Proton

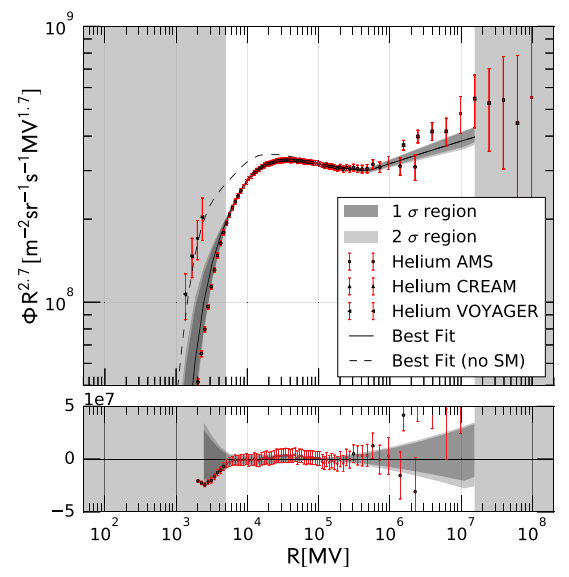

(b) Helium

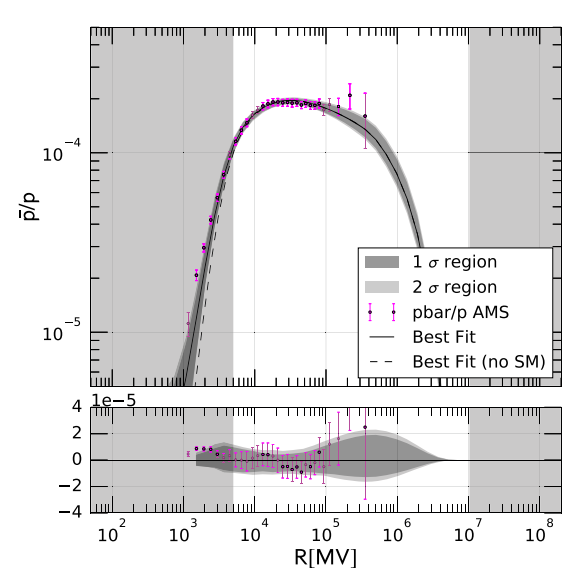

(c) $\bar{p} / p$

FIG. 13. Comparison between data and best-fit model for a diffusion model without convection and rigidity cut at $5 \mathrm{GV}$ (noVc-5 GV). 

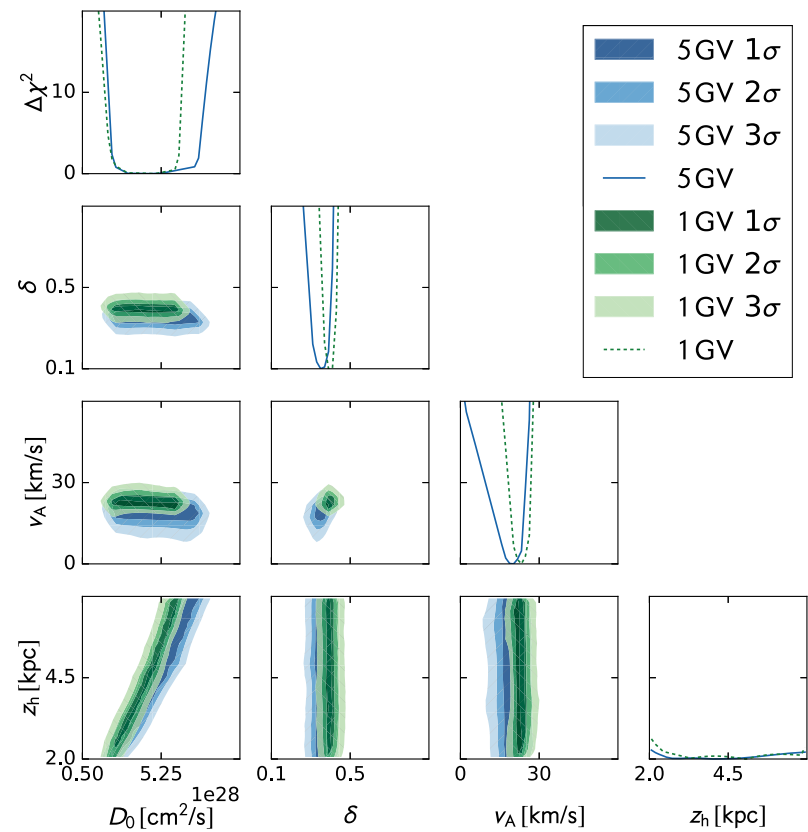

FIG. 14. Comparison of the propagation parameters in the case of no convection for a rigidity cutoff at $1 \mathrm{GV}$ (noVc-1 GV) and $5 \mathrm{GV}$ (noVc-5 GV).

necessary, complemented by the use of time series of CR data.

\section{F. Fit without convection}

As the last cross-check we investigate the necessity of convection. The baseline fit allows convection velocities and finds a tendency toward large values $\gtrsim 50 \mathrm{~km} / \mathrm{s}$, while the fit including data down to $1 \mathrm{GV}$ prefers low values. Therefore, we also test a diffusion model without convection [labeled (noVc-5 GV)] and compare it to our baseline fit. This also allows a more direct comparison with previous works where convection was not considered $[9,10]$. Results are shown in Figs. 12 and 13. The residuals in Fig. 13 show a flat behavior. The resulting $\chi^{2} / \mathrm{NDF}$ is $48.7 / 146$. On the other hand, the amount of solar modulation $340_{-125}^{+45} \mathrm{MV}$ appears to be too low. Figure 12 shows the comparison of the remaining propagation parameters to the baseline fit. The model without convection prefers slightly higher values for $\delta$, slightly lower values for $D_{0}$, and a well constrained value of $v_{\mathrm{A}}$, so that overall the results seem more in agreement with the baseline fit extending down to $1 \mathrm{GV}$, rather than the main case down to $5 \mathrm{GV}$. Given also the low value for the solar modulation in the case (noVc-5 GV) we thus also tested the case of data down to $1 \mathrm{GV}$ (labeled noVc-1 GV). In this case the $\chi^{2} / \mathrm{NDF}$ increases to $82.2 / 176$, but we achieve a more reasonable value for the solar modulation potential of $640 \pm 20 \mathrm{MV}$. The propagation parameters are not much affected by the data below $5 \mathrm{GV}$ as can be seen from Fig. 14. Figure 15 shows that residuals are reasonably flat also for this fit.

We report in Table III a summary of the $1 \sigma$ constraints on the parameters for the various fits performed. When the lower or upper range coincides with the chosen prior, the constraint is reported in italicized characters. The $\chi^{2}$ values for each fit, also broken into the sub-data set, used are reported in Table IV. We can use the results from Table III to derive the systematic uncertainties on $\delta$. Averaging between the (main) fit and the $(1 \mathrm{GV})$ fit we get a value of $\delta=0.30_{-0.02}^{+0.03}$. From the fits without convection we see that $\delta$ can be upshifted by up to a value of 0.1 , while in the (di Mauro) fits $\delta$ can be downshifted by a value of 0.04 . We thus quote these last two numbers as systematic uncertainties so that $\delta=0.30_{-0.02}^{+0.03}$ (stat $)_{-0.04}^{+0.10}$ (sys).

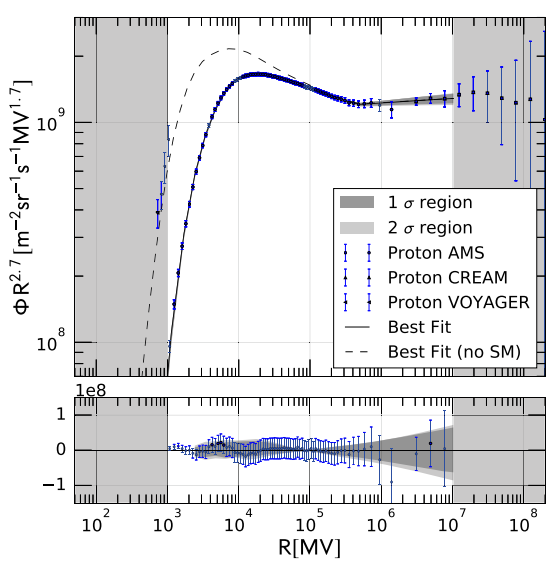

(a) Proton

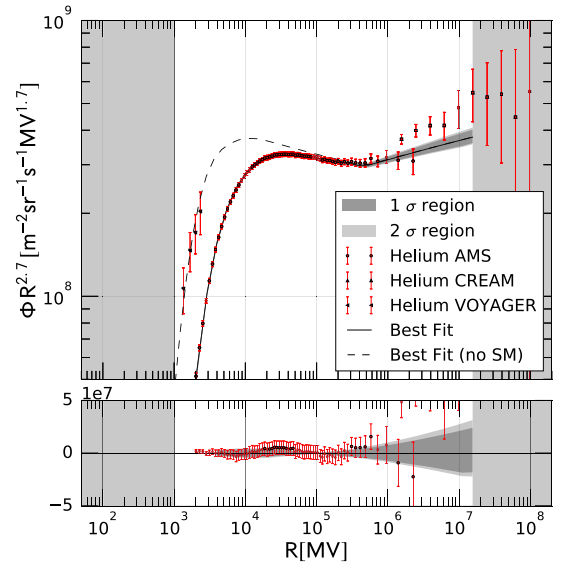

(b) Helium

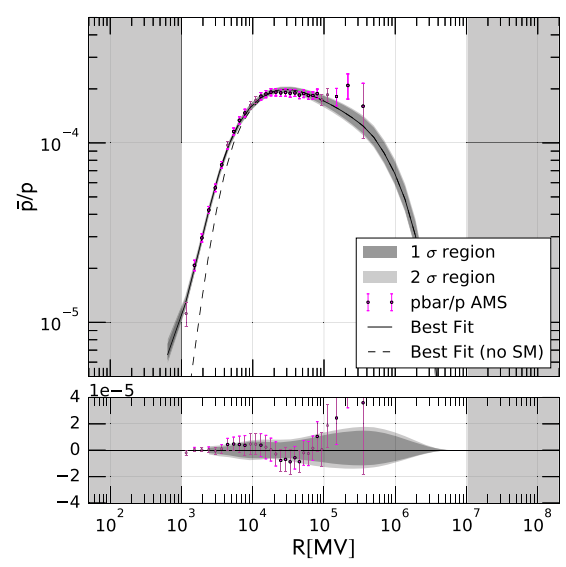

(c) $\bar{p} / p$

FIG. 15. Comparison between data and best-fit model for a diffusion model without convection and with data down to $1 \mathrm{GV}$ (noVc-1 GV). 


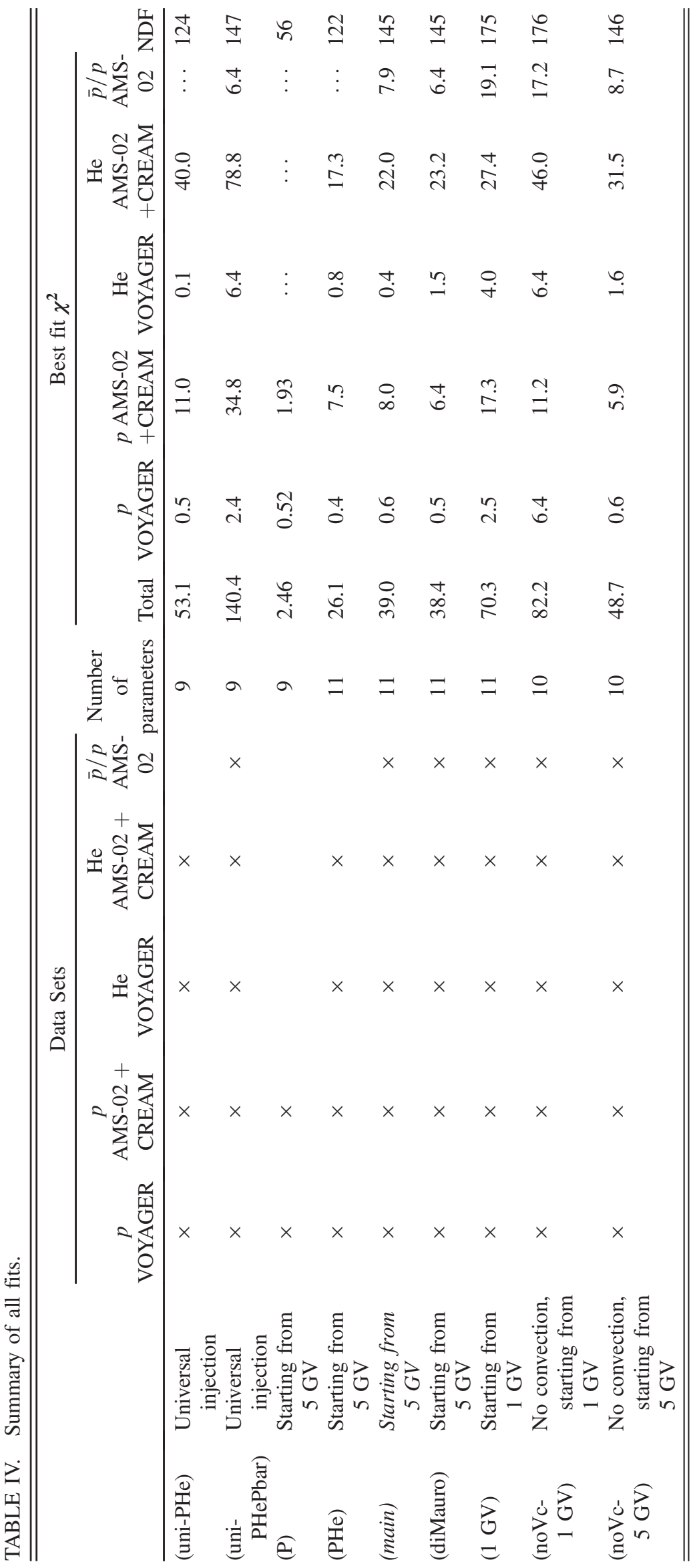




\section{SUMMARY AND CONCLUSION}

We have presented new constraints on the propagation of galactic CRs from an (up to) 11-dimensional parameter fit to the latest AMS-02 spectra for $p$, He, and $\bar{p} / p$. Solar modulation is treated within the force-field approximation, but the modulation potential is constrained with a novel approach, fitting the unmodulated CR $p$ and He spectra to recently available low-energy data from VOYAGER, collected after the probe left the heliosphere and thus sampling the local interstellar CR flux. The VOYAGER data and the unmodulated spectra are fitted jointly to the AMS-02 data and the modulated spectra.

As a first attempt, we try to fit the data with a universal injection spectrum for $p$ and He. We find that a universal injection is possible when fitting only $p$ and He data. In this case, the observed difference in $p$ and He slopes of about $\sim 0.1$ can be explained by a significant production of secondary $p$ so that the total primary plus secondary $p$ spectrum is steepened by the required 0.1 value in the slope. However, this requires a quite low value of the spectral index of diffusion $\delta \sim 0.15$ and implies a large production of $\bar{p}$ which significantly overpredicts the observations. This scenario is, thus, in the final instance, not viable. For the main results we thus perform a fit leaving individual spectral freedom to $p$ and He. With this additional freedom a good fit to $p, \mathrm{He}, \bar{p} / p$ spectra are achieved. The main result is a tight constraint on $\delta=0.30_{-0.02}^{+0.03}(\mathrm{stat})_{-0.04}^{+0.10}$ (sys), where the error is dominated by systematic uncertainties rather than statistical ones. The robustness of this result has been cross-checked against various factors, like the uncertainties in the solar modulation, the choice of the diffusion model framework, i.e., if convection is allowed or not, and the systematic uncertainties in the $\bar{p}$ production cross section. Since solar modulation is most important at low energies, its effect was studied using different cuts ( 1 and $5 \mathrm{GV})$ on the AMS02 data. The effect of uncertainties in the $\bar{p}$ production cross section was, instead, tested comparing the results of the fit when different available determinations of the cross section are used. Both of these effects have an order 10\%-20\% impact on the value of $\delta$, while the most important effect is the inclusion of convection in the model, which shifts the value of $\delta$ from $\sim 0.3$ to $\sim 0.4$.

For the other propagation parameters the results are less definitive. The height of the galactic halo and the normalization of diffusion present a well-known degeneracy, which, not surprisingly, cannot be resolved. In this respect, more precise "CR-clocks" measurements, like the ratio ${ }^{9} \mathrm{Be} /{ }^{10} \mathrm{Be}$, which will be available in the future from AMS-02, are necessary. Regarding convection and reacceleration, the fit above $5 \mathrm{GV}$ prefers large convection velocities of $v_{0, c} \gtrsim$ $50 \mathrm{~km} / \mathrm{s}$ and Alfven velocities of $v_{\mathrm{A}} \lesssim 25 \mathrm{~km} / \mathrm{s}$, with large parameter errors coming from a degeneracy between convection and reacceleration. The fit with data down to $1 \mathrm{GV}$ breaks this degeneracy and gives a well definite reacceleration of $v_{\mathrm{A}}=25 \pm 2 \mathrm{~km} / \mathrm{s}$ and preference for lower values of $v_{0, c} \lesssim 50 \mathrm{~km} / \mathrm{s}$. It remains, however, unclear how robust this determination of $v_{\mathrm{A}}$ is, since it relies on data below $5 \mathrm{GV}$ which are significantly affected by solar modulation. Finally, we find that a fit without convection is nonetheless possible, providing a good fit to the $p, \mathrm{He}$, and $\bar{p} / p$ data, and giving a similar value of $v_{\mathrm{A}}$.

A comparison of these results from the constraints imposed from the AMS-02 observations of lithium, boron, and carbon will be presented in a forthcoming companion paper.

\section{ACKNOWLEDGMENTS}

We wish to thank Pasquale Serpico for numerous useful discussions, in particular on antiproton cross sections. We thank Leila Ali Cavasonza, Jan Heisig, Michael Krämer, Julien Lesgourgues, and Andy Strong for helpful discussions and comments.
[1] O. Adriani et al., PAMELA measurements of cosmic-ray proton and helium spectra, Science 332, 69 (2011).

[2] M. Aguilar et al., Precision Measurement of the Proton Flux in Primary Cosmic Rays from Rigidity $1 \mathrm{GV}$ to $1.8 \mathrm{TV}$ with the Alpha Magnetic Spectrometer on the International Space Station, Phys. Rev. Lett. 114, 171103 (2015).

[3] M. Aguilar et al., Precision Measurement of the Helium Flux in Primary Cosmic Rays of Rigidities $1.9 \mathrm{GV}$ to $3 \mathrm{TV}$ with the Alpha Magnetic Spectrometer on the International Space Station, Phys. Rev. Lett. 115, 211101 (2015).

[4] A. E. Vladimirov, G. Johannesson, I. V. Moskalenko, and T. A. Porter, Testing the origin of high-energy cosmic rays, Astrophys. J. 752, 68 (2012).
[5] P. D. Serpico, Possible physics scenarios behind cosmic-ray "anomalies", arXiv:1509.04233.

[6] P. Blasi, E. Amato, and P. D. Serpico, Spectral Breaks as a Signature of Cosmic Ray Induced Turbulence in the Galaxy, Phys. Rev. Lett. 109, 061101 (2012).

[7] P. Blasi and E. Amato, Diffusive propagation of cosmic rays from supernova remnants in the Galaxy. I: Spectrum and chemical composition, J. Cosmol. Astropart. Phys. 01 (2012) 010.

[8] R. Aloisio, P. Blasi, and P. Serpico, Nonlinear cosmic ray Galactic transport in the light of AMS-02 and Voyager data, Astron. Astrophys. 583, A95 (2015). 
[9] R. Trotta, G. Johannesson, I. V. Moskalenko, T. A. Porter, R. Ruiz de Austri, and A. W. Strong, Constraints on cosmic-ray propagation models from a global Bayesian analysis, Astrophys. J. 729, 106 (2011).

[10] G. Johannesson et al., Bayesian analysis of cosmic-ray propagation: Evidence against homogeneous diffusion, Astrophys. J. 824, 16 (2016).

[11] G. Di Bernardo, C. Evoli, D. Gaggero, D. Grasso, and L. Maccione, Unified interpretation of cosmic-ray nuclei and antiproton recent measurements, Astropart. Phys. 34, 274 (2010).

[12] D. Maurin, F. Donato, R. Taillet, and P. Salati, Cosmic rays below $z=30$ in a diffusion model: New constraints on propagation parameters, Astrophys. J. 555, 585 (2001).

[13] A. Putze, L. Derome, and D. Maurin, A Markov Chain Monte Carlo technique to sample transport and source parameters of Galactic cosmic rays: II. Results for the diffusion model combining $\mathrm{B} / \mathrm{C}$ and radioactive nuclei, Astron. Astrophys. 516, A66 (2010).

[14] A. Kounine, Latest results from the alpha magnetic spectrometer: Positron fraction and antiproton/proton ratio, http://indico.cern.ch/event/381134/contributions/900587/ attachments/759339/1041605/AMS_positrons_antiprotons .pdf (accessed 05-04-2015).

[15] E. C. Stone, A. C. Cummings, F. B. McDonald, B. C. Heikkila, N. Lal, and W. R. Webber, Voyager 1 observes low-energy galactic cosmic rays in a region depleted of heliospheric ions, Science 341, 150 (2013).

[16] M. di Mauro, F. Donato, A. Goudelis, and P. D. Serpico, New evaluation of the antiproton production cross section for cosmic ray studies, Phys. Rev. D 90, 085017 (2014).

[17] Oscar Adriani et al., An anomalous positron abundance in cosmic rays with energies $1.5-100 \mathrm{GeV}$, Nature (London) 458, 607 (2009).

[18] M. Aguilar et al., First Result from the Alpha Magnetic Spectrometer on the International Space Station: Precision Measurement of the Positron Fraction in Primary Cosmic Rays of 0.5-350 GeV, Phys. Rev. Lett. 110, 141102 (2013).

[19] N. J. Shaviv, E. Nakar, and T. Piran, Natural explanation for the anomalous positron to electron ratio with supernova remnants as the sole cosmic ray source, Phys. Rev. Lett. 103, 111302 (2009).

[20] A. W. Strong, I. V. Moskalenko, and V. S. Ptuskin, Cosmicray propagation and interactions in the Galaxy, Annu. Rev. Nucl. Part. Sci. 57, 285 (2007).

[21] R. Blandford and D. Eichler, Particle acceleration at astrophysical shocks: A theory of cosmic ray origin, Phys. Rep. 154, 1 (1987).

[22] V. A. Dogiel, V. S. Berezinsky, S. V. Bulanov, and V. S. Ptuskin, Astrophysics of Cosmic Rays (North-Holland, Amsterdam, 1990).

[23] E.S. Seo and V.S. Ptuskin, Stochastic reacceleration of cosmic rays in the interstellar medium, Astrophys. J. 431, 705 (1994).

[24] A. Putze, L. Derome, D. Maurin, L. Perotto, and R. Taillet, A Markov Chain Monte Carlo for galactic cosmic rRay physics: I. Method and results for the leaky box model, Astron. Astrophys. 497, 991 (2009).
[25] C. Evoli, D. Gaggero, and D. Grasso, Secondary antiprotons as a galactic dark matter probe, J. Cosmol. Astropart. Phys. 12 (2015) 039.

[26] I. Yusifov and I. Kucuk, Revisiting the radial distribution of pulsars in the galaxy, Astron. Astrophys. 422, 545 (2004).

[27] G. L. Case and D. Bhattacharya, A new sigma-d relation and its application to the galactic supernova remnant distribution, Astrophys. J. 504, 761 (1998).

[28] D. A. Green, Constraints on the distribution of supernova remnants with galactocentric radius, Mon. Not. R. Astron. Soc. 454, 1517 (2015).

[29] I. V. Moskalenko, A. W. Strong, J. F. Ormes, and M. S. Potgieter, Secondary anti-protons and propagation of cosmic rays in the galaxy and heliosphere, Astrophys. J. 565, 280 (2002).

[30] A. W. Strong, I. V. Moskalenko, and O. Reimer, Diffuse continuum gamma-rays from the galaxy, Astrophys. J. 537, 763 (2000); 541, 1109(E) (2000).

[31] A. W. Strong, Recent extensions to GALPROP, arXiv: 1507.05020 .

[32] B. Coste, L. Derome, D. Maurin, and A. Putze, Constraining Galactic cosmic-ray parameters with $Z<=2$ nuclei, Astron. Astrophys. 539, A88 (2012).

[33] Y.S. Yoon et al., Cosmic-ray proton and helium spectra from the first CREAM flight, Astrophys. J. 728, 122 (2011).

[34] E. N. Parker, Cosmic-ray modulation by solar wind, Phys. Rev. 110, 1445 (1958).

[35] I. G. Usoskin, K. Alanko-Huotari, G. A. Kovaltsov, and K. Mursula, Heliospheric modulation of cosmic rays: Monthly reconstruction for 1951-2004, J. Geophys. Res. 110, A12108 (2005).

[36] L. J. Gleeson and W. I. Axford, Solar modulation of galactic cosmic rays, Astrophys. J. 154, 1011 (1968).

[37] L. A. Fisk, Solar modulation and a galactic origin for the anomalous component observed in low-energy cosmic rays, Astrophys. J. 206, 333 (1976).

[38] I. G. Usoskin, G. A. Bazilevskaya, and G. A. Kovaltsov, Solar modulation parameter for cosmic rays since 1936 reconstructed from ground-based neutron monitors and ionization chambers, J. Geophys. Res. 116, n/a (2011).

[39] I. Cholis, D. Hooper, and T. Linden, A predictive analytic model for the solar modulation of cosmic rays, Phys. Rev. D 93, 043016 (2016).

[40] M. Potgieter, Solar modulation of cosmic rays, Living Rev. Solar Phys. 10, 3 (2013).

[41] O. Adriani et al., Time dependence of the proton flux measured by PAMELA during the July 2006-December 2009 solar minimum, Astrophys. J. 765, 91 (2013).

[42] M. S. Potgieter, E. E. Vos, M. Boezio, N. De Simone, V. Di Felice, and V. Formato, Modulation of galactic protons in the heliosphere during the unusual solar minimum of 2006 to 2009, Sol. Phys. 289, 391 (2014).

[43] C. Corti, V. Bindi, C. Consolandi, and K. Whitman, Solar modulation of the proton local interstellar spectrum with AMS-02, Voyager 1, and PAMELA, Astrophys. J. 829, 1 (2016).

[44] A. Ghelfi, D. Maurin, A. Cheminet, L. Derome, G. Hubert, and F. Melot Neutron monitors and muon detectors for solar modulation studies: 2. $\phi$ time series. 2016. DOI: 10.1016/ j.asr.2016.06.027 (2016). 
[45] F. Feroz, M. P. Hobson, and M. Bridges, MultiNest: An efficient and robust Bayesian inference tool for cosmology and particle physics, Mon. Not. R. Astron. Soc. 398, 1601 (2009).

[46] J. Skilling, Nested sampling for general Bayesian computation, 2006, http://www.mrao.cam.ac.uk/steve/maxent2009/ images/skilling.pdf (accessed 05-04-2015).

[47] W. A. Rolke, A. M. Lopez, and J. Conrad, Limits and confidence intervals in the presence of nuisance parameters, Nucl. Instrum. Methods Phys. Res., Sect. A 551, 493 (2005).

[48] M. A. Malkov, P. H. Diamond, and R. Z. Sagdeev, Protonhelium Spectral Anomaly as a Signature of Cosmic Ray Accelerator, Phys. Rev. Lett. 108, 081104 (2012).

[49] Y. Ohira, N. Kawanaka, and K. Ioka, Cosmic-ray hardenings in light of AMS-02 data, Phys. Rev. D 93, 083001 (2016).

[50] Y. Ohira and K. Ioka, Cosmic-ray helium hardening, Astrophys. J. Lett. 729, L13 (2011).
[51] L. O'C. Drury and A. W. Strong, Cosmic-ray diffusive reacceleration: A critical look, Proc. Sci., ICRC2015 (2015) 483 [arXiv:1508.02675].

[52] F. Donato, D. Maurin, P. Salati, A. Barrau, G. Boudoul, and R. Taillet, Anti-protons from spallations of cosmic rays on interstellar matter, Astrophys. J. 563, 172 (2001).

[53] H. G. Fischer, Baryon yields, isospin effects and strangeness production in elementary hadronic interactions, Heavy Ion Phys. 17, 369 (2003).

[54] R. Kappl and M. W. Winkler, The cosmic ray antiproton background for AMS-02, J. Cosmol. Astropart. Phys. 14 (2014) 051.

[55] L. C. Tan and L. K. Ng, Calculation of the equilibrium anti-proton spectrum, J. Phys. G 9, 227 (1983).

[56] M. Simon, A. Molnar, and S. Roesler, A new calculation of the interstellar secondary cosmic-ray antiprotons, Astrophys. J. 499, 250 (1998). 